\title{
COVERS OF GENERALIZED QUADRANGLES, 2. KANTOR-KNUTH COVERS AND EMBEDDED OVOIDS
}

\author{
JOSEPH A. THAS AND KOEN THAS
}

\begin{abstract}
In this paper, which is a sequel to [14], we proceed with our study of covers and decomposition laws for geometries related to generalized quadrangles. In particular, we obtain a higher decomposition law for all KantorKnuth generalized quadrangles which generalizes one of the main results in [14]. In a second part of the paper, we study the set of all Kantor-Knuth ovoids (with given parameter) in a fixed finite parabolic quadrangle, and relate this set to embeddings of parabolic quadrangles into Kantor-Knuth quadrangles. This point of view gives rise to an answer of a question posed in [13].
\end{abstract}

CONTENTS

$\begin{array}{lr}\text { 1. Introduction } & 1 \\ \text { 2. } & \text { Basic geometric notions } \\ \text { 3. Higher and lower decomposition } & 2 \\ \text { 4. Basic properties of Kantor-Knuth quadrangles and Kantor-Knuth ovoids } & 6 \\ \text { 5. Synopsis of translation ovoids } & 6 \\ \text { 6. Decomposition laws for Kantor-Knuth generalized quadrangles } \\ \text { 7. Embedded Kantor-Knuth ovoids } \\ \text { 8. The automorphism group via the good orbit? } \\ \text { References }\end{array}$

\section{INTRODUCTION}

1.1. Decomposition laws. In the paper [3], Cardinali and Sastry study various aspects of geometries related to ovoids of the classical finite symplectic generalized quadrangle $\mathcal{W}\left(2^{n}\right)$. In particular, starting with the natural embedding $\mathcal{W}\left(2^{n}\right) \hookrightarrow \mathcal{Q}\left(5,2^{n}\right)$, they consider the geometry $\mathcal{A}$ defined by $\mathcal{Q}\left(5,2^{n}\right) \backslash \mathcal{W}\left(2^{n}\right)$, and the geometry $\mathcal{E}$ which consists of elliptic ovoids in $\mathcal{W}\left(2^{n}\right)$ as points, and rosettes of such ovoids as lines (details related to these notions can be found in the next two sections). There is a natural projection

$$
\pi: \mathcal{A} \mapsto \mathcal{E} .
$$

Cardinali and Sastry ask if any given 2-cover $\gamma: \mathcal{A} \mapsto \mathcal{E}$ factors through $\pi$ and an automorphism $\widetilde{\alpha}$ of $\mathcal{A}$ (or $\left.Q\left(5,2^{n}\right)\right)$ :

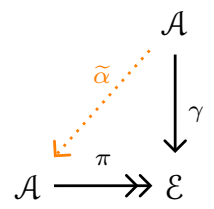

2000 Mathematics Subject Classification. 05B25; 05E18; 51A10; 51B25; 51E12.

Key words and phrases. cover; generalized quadrangle; morphism; semipartial geometry; subtended ovoid. 
That question was the starting point of [14], and in a more general setting - without finiteness restrictions, and without assuming that the quadrangles in question are classical - a complete answer was provided in loc. cit.

More precisely, we have:

Theorem 1.1 ([14]). Let $\mathcal{S}$ be a thick generalized quadrangle, and let $\mathcal{S}^{\prime} \hookrightarrow \mathcal{S}$ be a thick full subquadrangle which is a geometrical hyperplane of $\mathcal{S}$. Define $\mathcal{A}$ and $\mathcal{E}$ as above, and let $\pi: \mathcal{A} \mapsto \mathcal{E}$ be the natural projection. Then every cover $\gamma: \mathcal{A} \mapsto \mathcal{E}$ factorizes as $\gamma=\pi \circ \widetilde{\alpha}$, with $\widetilde{\alpha}$ an automorphism of $\mathcal{A}$, if and only if every automorphism of $\mathcal{E}$ is induced by an automorphism of $\mathcal{S}$.

In [14], we called the factorization $\gamma=\pi \circ \widetilde{\alpha}$ "higher decomposition," and in [14], other factorizations were studied (cf. section 3 for more details). Plugging in the data $\left(\mathcal{S}, \mathcal{S}^{\prime}\right)=(\mathcal{Q}(5, q), \mathcal{W}(q))$ in Theorem 1.1 readily leads to the answer of the question of Cardinali and Sastry for all prime powers $q$.

The first main result of the present paper yields higher decomposition for the class of finite Kantor-Knuth quadrangles (with parabolic subquadrangles). For $q$ an odd prime power, this generalizes our answer to the question of Cardinali and Sastry, since $2(5, q)$ is just a classical Kantor-Knuth generalized quadrangle.

In particular, we will obtain the following result in section 6 (where $\mathcal{A}$ and $\mathcal{E}$ are constructed from a nonclassical Kantor-Knuth quadrangle with a full parabolic subquadrangle):

Theorem 1.2. Let $\gamma: \mathcal{A} \longmapsto \mathcal{E}$ be a 2-cover of $\mathcal{E}$. Then $\gamma$ induces an automorphism $\bar{\alpha}$ of $\mathcal{S}^{\prime}$ and there exist exactly two automorphisms $\widetilde{\alpha}$ of $\mathcal{S}$ which extend the automorphism $\bar{\alpha}$ of $\mathcal{S}^{\prime}$.

1.2. A garden of Kantor-Knuth ovoids. The starting point of the second part of this paper is the following quote (taken from [13], pp. 245):

"Note that the number $q^{2}\left(q^{2}-1\right)\left(q^{4}-1\right) / 4$ of ovoids produced for $q$ a power of 3 is much larger than the number of points $z \in \mathcal{S} \backslash \mathcal{S}_{\gamma}$ available to be used in the original construction."

Indeed, although Kantor-Knuth ovoids are initially defined as subtended ovoids $\mathcal{O}_{x}$ in parabolic quadrangles $\mathcal{Q}(4, q)$ which are full subquadrangles in Kantor-Knuth quadrangles, a calculation shows that the $\operatorname{Aut}(\mathcal{Q}(4, q))$ orbit of $\mathcal{O}_{x}$ contains much more elements than the number of ovoids obtained as subtended ovoids. In section:7, we try to understand this difference, by observing that one can partition the orbit in certain equivalence classes, each class of which corresponds to an embedding of $Q(4, q)$ in a Kantor-Knuth quadrangle (of the same type as the Kantor-Knuth ovoid).

In order to facilitate this view, we study a category of embeddings of parabolic quadrangles in Kantor-Knuth quadrangles in much detail, and meet a number of interesting combinatorial problems related to covers on the wayside.

1.3. Organization. In section 2, we sum up some basic notions concerning the geometry of generalized quadrangles (and related geometries). In section 3, we recall higher and lower decomposition from [14]. Sections 4 and 5 provide a short overview of the basic properties of Kantor-Knuth quadrangles, Kantor-Knuth ovoids and translation ovoids which we will need in our paper. Then, in section 6 , higher decomposition is obtained for all Kantor-Knuth quadrangles. In section 7 we study embeddings of parabolic quadrangles in Kantor-Knuth quadrangles and the various related combinatorial problems which arise. Finally, in the last section, we calculate which fraction of the size of the automorphism group of a Kantor-Knuth ovoid is induced by the automorphism group of a Kantor-Knuth quadrangle.

Our approach in this paper is mainly synthetic.

\section{BASIC GEOMETRIC NOTIONS}

We summarize some basic notions that will be used throughout. Standard references are [5, 10, 15]. 
2.1. Quadrangles. A generalized quadrangle (GQ) of order $(s, t)$, where $s$ and $t$ are cardinal numbers, is a point-line incidence geometry with the following properties:

(a) Each point is incident with $t+1$ lines and each line is incident with $s+1$ points.

(b) If $(x, L)$ is a non-incident point-line pair, there is precisely one point-line pair $(y, M)$ such that $x \mathbf{I} M \mathbf{I} y \mathbf{I} L$ (here, "I" denotes the incidence relation).

(c) Two distinct points are incident with at most one line.

If both $s$ and $t$ are at least 2, we say that the GQ is thick; otherwise it is called thin.

We use the usual notation $x \sim y$ to indicate that the points $x$ and $y$ are collinear, and any point is collinear with itself. For any point $x, x^{\perp}:=\{z \mid z \sim x\}$, and for any subset $Y$ of the point set, $Y^{\perp}:=\cap_{y \in Y} y^{\perp}$; we make no distinction between $y^{\perp}$ and $\{y\}^{\perp}$. In particular, we denote $\left(Y^{\perp}\right)^{\perp}$ by $Y^{\perp \perp}$.

Finally, if $u$ and $v$ are distinct points, then $\operatorname{cl}(u, v)$ is the point set $\left\{w \mid w^{\perp} \cap\{u, v\}^{\perp \perp} \neq \emptyset\right\}$.

2.2. Subquadrangles. If $\mathcal{S}=(\mathcal{P}, \mathcal{L}, \mathbf{I})$ is a generalized quadrangle (where $\mathcal{P}$ is the point set, $\mathcal{L}$ the line set, and I is incidence), then a subgeometry is a triple $\Gamma=\left(\mathcal{P}^{\prime}, \mathcal{L}^{\prime}, \mathbf{I}^{\prime}\right)$ such that $\mathcal{P}^{\prime} \subseteq \mathcal{P}, \mathcal{L}^{\prime} \subseteq \mathcal{L}$, and $\mathbf{I}^{\prime}$ is the induced incidence relation.

A subquadrangle (subGQ) is a subgeometry which is a generalized quadrangle. A subquadrangle $\mathcal{S}^{\prime}$ of a generalized quadrangle $\mathcal{S}$ is full if for all its lines $U$, we have the property that any point of $\mathcal{S}$ incident with $U$ is also a point of $\mathcal{S}^{\prime}$. Dually, we speak of "ideal subquadrangles."

2.3. Ovoids. If $\mathcal{S}$ is a generalized quadrangle, an ovoid of $\mathcal{S}$ is a set of points $\mathcal{O}$ in $\mathcal{S}$ such that each line contains precisely one point of $\mathcal{O}$.

2.4. Semi partial geometries. A (finite) semi partial geometry is an incidence structure $\mathcal{J}=(\mathcal{P}, \mathcal{B}, I)$, in which $\mathcal{P}$ and $\mathcal{B}$ are disjoint, non-empty sets of points and lines, for which $\mathrm{I}$ is a symmetric point-line incidence relation satisfying the following axioms:

(i) each point is incident with $1+t^{*}$ lines, with $t^{*} \geq 1$, and two distinct points are incident with at most one line;

(ii) each line is incident with $1+s^{*}$ points, with $s^{*} \geq 1$, and two distinct lines are incident with at most one point;

(iii) if a point $x$ and a line $L$ are not incident, then there are 0 or $\alpha^{*}$ points, where $\alpha^{*} \geq 1$, which are collinear with $x$ and incident with $L$;

(iv) if two points are not collinear, then there are $\mu^{*}$ points, where $\mu^{*}>0$, collinear with both.

The integers $s^{*}, t^{*}, \alpha^{*}, \mu^{*}$ are the parameters of the semi partial geometry. The semi partial geometries with $\alpha^{*}=1$ are the partial quadrangles. A semi partial geometry is a partial geometry if and only if the 0 in Axiom (iii) does not occur; this is equivalent to the condition $\mu^{*}=\left(t^{*}+1\right) \alpha^{*}$.

A generalized quadrangle is a partial geometry with $\alpha^{*}=1$, so $\mu^{*}=t^{*}+1$.

For more on semi partial geometries we refer to [5].

2.5. Morphisms. If $\Gamma=(\mathcal{P}, \mathcal{L}, \mathbf{I})$ and $\Gamma^{\prime}=\left(\mathcal{P}^{\prime}, \mathcal{L}^{\prime}, \mathbf{I}^{\prime}\right)$ are point-line incidence geometries, then a morphism $\gamma: \Gamma \mapsto \Gamma^{\prime}$ is a map from $\mathcal{P} \cup \mathcal{L}$ to $\mathcal{P}^{\prime} \cup \mathcal{L}^{\prime}$ which sends points to points, lines to lines, and preserves incidence. If $\gamma$ is bijective and the inverse map also preserves incidence, we say $\gamma$ is an isomorphism. If $\Gamma=\Gamma^{\prime}$, an isomorphism is also called an automorphism, and the set of automorphisms of $\Gamma$ naturally forms a group under composition of maps, which is denoted by $\operatorname{Aut}(\Gamma)$.

\section{HigHER AND LOWER DECOMPOSITION}

In this section, we describe higher and lower decomposition as introduced in [14]. 
3.1. $\mathcal{A}$ and $\mathcal{E}$. Let $\mathcal{S}$ be a thick generalized quadrangle, and let $\mathcal{S}^{\prime}$ be a thick full subquadrangle of $\mathcal{S}$. Let $x$ be a point of $\mathcal{S} \backslash \mathcal{S}^{\prime}$. Then $x^{\perp} \cap \mathcal{S}^{\prime}=\mathcal{O}_{x}$ is easily seen to be an ovoid of $\mathcal{S}^{\prime}$, which we call a subtended ovoid (by $x$ ). Let $L$ be any line of $\mathcal{S} \backslash \mathcal{S}^{\prime}$ which meets $\mathcal{S}^{\prime}$ in a point $l$. Then each point of $L \backslash\{l\}$ subtends an ovoid in $\mathcal{S}^{\prime}$, and the set of all these ovoids is called the rosette $R_{L}$ of ovoids determined by $L$. If $\mathcal{S}^{\prime}$ is also a geometrical hyperplane, there is a natural bijection between the lines incident with $x$ and the points of $x^{\perp} \cap \mathcal{S}^{\prime}$.

Keeping the latter hypothesis, we define the geometry $\mathcal{E}$ to have as points the subtended ovoids in $\mathcal{S}^{\prime}$, and as lines the rosettes $R_{L}$. Incidence is symmetrized containment. We also define a geometry $\mathcal{A}$, which is just the affine generalized quadrangle which arises when taking away the geometric hyperplane $\mathcal{S}^{\prime}$ of $\mathcal{S}$.

Note that there is a natural projection

$$
\pi: \mathcal{A} \longmapsto \mathcal{E},\left\{\begin{aligned}
x \longmapsto \mathcal{O}_{x} & \text { for all points } x \\
L \longmapsto R_{L} & \text { for all lines } L .
\end{aligned}\right.
$$

By the mere definition of $\mathcal{E}, \pi$ is surjective on both points and lines.

3.2. Covers. Let $\gamma: \Gamma \longmapsto \Gamma^{\prime}$ be a morphism between point-line incidence geometries. Suppose both geometries are not empty - so they have either at least one point or at least one line. (In fact, since $\gamma$ is a map, it suffices to ask that $\Gamma$ is not empty.) Then $\gamma$ is a cover if $\gamma$ is locally a bijection, that is, if $x$ is any point of $\Gamma, \gamma$ induces a bijection between the lines incident with $x$ and the lines incident with $\gamma(x)$, and if $L$ is a line of $\Gamma$, it induces a bijection between the points incident with $L$ and the points incident with $\gamma(L)$. Sometimes we also say that $(\Gamma, \gamma)$ is a cover (of $\Gamma^{\prime}$ ), or even that $\Gamma$ is a cover of $\Gamma^{\prime}$ if the covering map is clear.

If $\Gamma^{\prime}$ is connected, then a cover $\gamma: \Gamma \longmapsto \Gamma^{\prime}$ is necessarily surjective; see [14].

If $\gamma: \Gamma \longmapsto \Gamma^{\prime}$ is a cover, and each fiber (of lines and points) has constant size $\theta$, we say that $\gamma$, or $(\Gamma, \gamma)$, or $\Gamma$ is a $\theta$-fold cover (of $\Gamma^{\prime}$ ) or simply a $\theta$-cover (of $\Gamma^{\prime}$ ). We also say that $\Gamma^{\prime}$ is $\theta$-covered by $\Gamma$.

\subsection{Factorization of morphisms I - Lower decomposition. The following theorem was proved in [14].}

Theorem 3.1 (Lower decomposition). Let $\mathcal{S}$ be a thick generalized quadrangle, and let $\mathcal{S}^{\prime}$ be a thick full subquadrangle which is a geometric hyperplane of $\mathcal{S}$. Define $\mathcal{A}$ and $\mathcal{E}$ as above, and let $\pi: \mathcal{A} \longmapsto \mathcal{E}$ be the natural projection. Then any cover $\gamma: \mathcal{A} \longmapsto \mathcal{E}$ factorizes as $\gamma=\alpha \circ \pi$, with $\alpha$ an automorphism of $\mathcal{E}$.

3.4. The automorphism $\bar{\alpha}$. Use the notation of the previous section. The following is taken from [14].

For each line $L$ of $\mathcal{E}$ the lines of $\gamma^{-1}(L)$ all contain the same point $u$ of $\mathcal{S}^{\prime}$. If the ovoids of the rosette $L$ all share the point $u^{\prime}$, then for any other rosette $M \neq L$ whose ovoids share $u^{\prime}$, the lines of $\gamma^{-1}(M)$ contain $u$. If $u^{\prime}=\zeta(u)$, and we let $u$ vary, we obtain a permutation

$$
\zeta: w \longmapsto w^{\prime}
$$

of the points of $\mathcal{S}^{\prime}$. This map $\zeta$ defines an automorphism $\bar{\alpha}$ of $\mathcal{S}^{\prime}$.

3.5. Factorization of morphisms II - Higher decomposition. We keep using the notation of the previous sections.

We say that $\pi: \mathcal{A} \longmapsto \mathcal{E}$ has the higher decomposition property if any cover $\gamma: \mathcal{A} \longmapsto \mathcal{E}$ factorizes as $\gamma=\pi \circ \widetilde{\alpha}$ for some $\widetilde{\alpha} \in \operatorname{Aut}(\mathcal{S})_{\mathcal{S}^{\prime}}$. We also say that $\gamma: \mathcal{A} \longmapsto \mathcal{E}$ has the higher decomposition property if it factorizes as above. Such an automorphism $\widetilde{\alpha}$ extends the automorphism $\bar{\alpha}$ of $\mathcal{S}^{\prime}$.

Theorem 3.2. The cover $\pi: \mathcal{A} \longmapsto \mathcal{E}$ has the higher decomposition property if and only if any automorphism of $\mathcal{E}$ is induced by an automorphism of $\mathcal{S}$.

Note that any automorphism of $\mathcal{E}$ is induced by an automorphism of $\mathcal{S}^{\prime}$. If every automorphism of $\mathcal{S}^{\prime}$ extends to an automorphism of $\mathcal{S}$, then every automorphism of $\mathcal{S}^{\prime}$ induces an automorphism of $\mathcal{E}$ in a faithful manner. In such a case higher decomposition is possible.

\section{Examples}


(1) Consider a subquadrangle $\mathcal{S}^{\prime} \cong \mathcal{Q}(4, q)$ of the generalized quadrangle $\mathcal{Q}(5, q)=\mathcal{S}$. Here the geometry $\mathcal{E}$ is 2-covered by $\mathcal{S} \backslash \mathcal{S}^{\prime}=\mathcal{A}$. As every automorphism of $\mathcal{S}^{\prime}$ extends to an automorphism of $\mathcal{S}$, higher decomposition is possible.

(2) Consider a subquadrangle $\mathcal{S}^{\prime} \cong \mathcal{H}\left(3, q^{2}\right)$ of the generalized quadrangle $\mathcal{H}\left(4, q^{2}\right)=\mathcal{S}$. Here the geometry $\mathcal{E}$ is $(q+1)$-covered by $\mathcal{S} \backslash \mathcal{S}^{\prime}=\mathcal{A}$. As every automorphism of $\mathcal{S}^{\prime}$ extends to an automorphism of $\mathcal{S}$, higher decomposition is possible.

(3) Consider a subquadrangle $\mathcal{S}^{\prime} \cong \mathcal{Q}(3, q)$ of the generalized quadrangle $Q(4, q)=\mathcal{S}$. Here the geometry $\mathcal{E}$ is $\theta$-covered by $\mathcal{S} \backslash \mathcal{S}^{\prime}=\mathcal{A}$, with $\theta=2$ for $q$ odd and $\theta=1$ for $q$ even. For $\theta=1$ it is clear that higher decomposition is possible. In the odd case not every automorphism of $\mathcal{S}^{\prime}$ extends to an automorphism of $\mathcal{S}$. But by Theorem 10.3 of [14] also here higher decomposition is possible.

(4) It is well known that the generalized quadrangle of order $\left(q, q^{2}\right)$ arising from a Kantor-Knuth flock [15] has subquadrangles of order $q$ isomorphic to $Q(4, q)$. For some of these subquadrangles the geometry $\mathcal{E}$ is 2 -covered by $\mathcal{A}$; for other subquadrangles $\mathcal{E}$ is 1 -covered by $\mathcal{A}$. In Section 6 higher decomposition in the 2-covered case will be considered.

3.6. Semi partial geometries and covers. As will be briefly explained in the next theorem, some $\theta$-covers give rise to semi partial geometries.

Theorem 3.3 ([14] ). Let $\mathcal{S}$ be a generalized quadrangle of order $(s, t)$ and let $\mathcal{S}^{\prime}$ be a subquadrangle of order $\left(s, t^{\prime}\right)$. If every subtended ovoid $\mathcal{O}_{x}$ of $\mathcal{S}^{\prime}$ is $\theta$-subtended, $\theta>1$, with $t^{\prime} \neq 1, t=s t^{\prime}$ and $(\theta-1) t=s^{2}$, then the geometry $\mathcal{E}$ is a semi partial geometry with parameters

$$
s^{*}=s-1, t^{*}=t, \alpha^{*}=\theta, \mu^{*}=\theta\left(t-t^{\prime}\right) .
$$

Examples.

(1) Considering Examples (1) and (4), with $\theta=2$, of section 3.5, there arises semi partial geometries with parameters

$$
s^{*}=q-1, t^{*}=q^{2}, \alpha^{*}=2, \mu^{*}=2 q(q-1) .
$$

(2) Considering Example (2), with $\theta=q+1$, of section 3.5, there arises a semi partial geometry with parameters

$$
s^{*}=q^{2}-1, t^{*}=q^{3}, \alpha^{*}=q+1, \mu^{*}=q(q+1)\left(q^{2}-1\right) .
$$

Remark 3.4. For the geometries described in the previous examples, we also refer to [2] and [5].

\section{BASIC PROPERTIES OF KANTOR-KNUTH QUADRANGLES AND KANTOR-KNUTH OVOIDS}

Let $\mathbb{F}_{q}$ be a finite field with $q$ odd, and let $\sigma \in \operatorname{Aut}\left(\mathbb{F}_{q}\right)$. Let $m$ be a given nonsquare in $\mathbb{F}_{q}$. Then one constructs a generalized quadrangle $\Gamma(q, \sigma)$ of order $\left(q, q^{2}\right)$, called Kantor-Knuth generalized quadrangle, as in [15, $\S 4.5$ and §5.7]. As our notation suggests, this construction is independent of the choice of $m$. On the other hand, two Kantor-Knuth GQs $\Gamma(q, \sigma)$ and $\Gamma\left(q, \sigma^{\prime}\right)$ are isomorphic if and only if $\sigma=\sigma^{\prime}$. (See [15] and the references therein.)

In this section, $\Gamma=\Gamma(q, \sigma)$ is a nonclassical Kantor-Knuth GQ of order $\left(q, q^{2}\right)$, where $q=p^{h}$ and $p$ is an odd prime.

4.1. The orbits $\Omega_{1}$ and $\Omega_{2}$. Let $\Omega$ be the set of all $q^{3}+q^{2}$ subGQs of order $q$ of $\Gamma$; all these subGQs are isomorphic to $Q(4, q)$ (see chapter 5 of [15], especially Theorem 5.1.9). In its action of $\operatorname{Aut}(\Gamma)$ on $\Omega$, there are two orbits $\Omega_{1}$ and $\Omega_{2}$, the first one having size $2 q^{2}$ and the latter having size $(q-1) q^{2}$.

Two more facts will be used without further notice:

- $\operatorname{Aut}(\Gamma)$ fixes some line which we denote by $[\infty]$, and which is contained in each element of $\Omega$ (by [16], there is a line $[\infty]$ each point of which is a translation point, and if this line would not be fixed by $\operatorname{Aut}(\Gamma)$, then each point of $\Gamma$ would be a translation point; in that case, it is well known that $\Gamma$ is classical [21]); 
- Aut $(\Gamma)$ acts transitively on the $q^{4}$ subGQs of order $(q, 1)$ containing [ $\infty$ ]. This can easily be derived from the fact that each point incident with $[\infty]$ is a translation point (see [16]), or equivalently that each line in $[\infty]^{\perp}$ is an axis of symmetry; $\operatorname{Aut}(\Gamma)$ acts transitively on the line pairs $(U, V)$ for which $U, V \in[\infty]^{\perp}$ and $U \nsim V$.

The latter fact will be especially useful in several calculations involving orbits which we will carry out below. Other properties will be mentioned in due course.

4.2. The automorphism group. The size of $\operatorname{Aut}(\Gamma)$ is $(q+1)(q-1)^{2} q^{6} \cdot \delta h$, where $\delta=4$ if $\sigma^{2}=1$ and $\sigma \neq 1$, and $\delta=2$ if $\sigma^{2} \neq 1[9]$.

4.3. Subtended ovoids. Let $\mathcal{Q} \in \Omega_{1}$ and let $x$ be a point of $\Gamma$ which is not a point of $\mathcal{Q}$; then each ovoid $\mathcal{O}_{x}$ in $Q$ is doubly subtended [14, Theorem 11.1]; as such, there arise $(q+1) q^{2}(q-1) / 2$ subtended ovoids. This fact is not true for subGQs coming from the orbit $\Omega_{2}$; there, each subtended ovoid is 1-subtended - see [14, Theorem 11.1] — and so we obtain $(q+1) q^{2}(q-1)$ such ovoids.

Call an ovoid arising from a point $x$ and a GQ in $\Omega$ a "Kantor-Knuth ovoid." From the facts below, this notion is well defined, independent of the choice of GQ-orbit:

FA let $(Q, x)$ and $\left(Q^{\prime}, x^{\prime}\right)$ be subGQ-point pairs of $\Gamma$ such that the subGQ is an element of $\Omega_{1}$, and the point is not contained in the subGQ; then by Thas [16], there is an automorphism $\alpha$ of $\Gamma$ which sends $Q$ to $Q^{\prime}$ and $x$ to $x^{\prime}$; in particular, $\alpha$ sends $\mathcal{O}_{x}$ (in Q) to $\mathcal{O}_{x^{\prime}}^{\prime}$ (in $Q^{\prime}$ ); the same statement is true for the orbit $\Omega_{2}$ (we will also obtain this result in 87.4 ;

FB by the previous point, by putting $\mathcal{Q}=Q^{\prime}$ (with $\mathcal{Q} \in \Omega$ ), we conclude that $\operatorname{Aut}(\Gamma)_{\mathcal{Q}}$ acts transitively on the points of $\Gamma$ outside $Q$.

By these facts, each subtended ovoid obtained from a subGQ in $\Omega$, and an exterior point (to the subGQ), merits the property of being named "Kantor-Knuth ovoid."

\section{SYNOPSIS OF TRANSLATION OVOIDS}

An ovoid $\mathcal{O}$ of $Q(4, q)$ is a translation ovoid with respect to the point $\omega \in \mathcal{O}$ if there is a point $\omega \in \mathcal{O}$ such that there is a subgroup $T$ of $\operatorname{Aut}(\mathcal{Q}(4, q))_{\mathcal{O}}$ which fixes $\omega$ linewise, and which acts sharply transitively on the points of $\mathcal{O} \backslash\{\omega\}$. Equivalent definitions can be found in [1].

If $\operatorname{Aut}(\mathcal{Q}(4, q))_{\mathcal{O}}$ does not fix $\omega$, it acts 2 -transitively on the points of $\mathcal{O}$, and then it is an elliptic quadric in $\mathcal{Q}(4, q)$ in case $q$ is odd (see e. g. [7]).

If $\Gamma$ is a semifield flock TGQ of order $\left(q, q^{2}\right)$, and $Q$ is a $Q(4, q)$-subGQ of $\Gamma$, then it can be shown that any subtended ovoid in $Q$ is a translation ovoid. Easy way to see this: if $\Gamma$ is classical, then such ovoids are elliptic quadrics; if $\Gamma$ is not classical, then there is a unique line [o] of translation points [16], and this is a line of 2 . Let $e$ be any point of $\Gamma$ not contained in $Q$, and let $e^{\prime}$ be the point incident with $[\infty]$ which is collinear with $e$. Let $T\left(e^{\prime}\right)$ be the translation group of $\Gamma$ with translation point $e^{\prime}$. For any two points $x, y$ in $\mathcal{O}_{e}$, supposed to be not incident with $[\infty]$, there is precisely one element in $T\left(e^{\prime}\right)$ which maps $x$ to $y$. It is obvious that this element stabilizes 2 , and so also $\mathcal{O}_{e}$. It is now easy to see that $\mathcal{O}_{e}$ is a translation ovoid relative to the point $e^{\prime}$, and that $T\left(e^{\prime}\right)_{e}$ acts (sharply) transitively on $\mathcal{O} \backslash\left\{e^{\prime}\right\}$.

\section{DECOMPOSITION LAWS FOR KANTOR-KNUTH GENERALIZED QUADRANGLES}

Consider a thick generalized quadrangle $\mathcal{S}$ of order $\left(s, s^{2}\right)$ satisfying the following conditions:

(i) $\mathcal{S}$ has a line $L$ of translation points;

(ii) $\mathcal{S}$ has a subquadrangle $\mathcal{S}^{\prime}$ of order $s$ containing $L$;

(iii) every subtended ovoid in $\mathcal{S}^{\prime}$ is doubly subtended.

By section 3.6 we know that the corresponding geometry $\mathcal{E}$ is a semi partial geometry with parameters

$$
s^{*}=s-1, t^{*}=s^{2}, \alpha^{*}=2, \mu^{*}=2 s(s-1) .
$$


Theorem 6.1. Let $\gamma: \mathcal{A} \longmapsto \mathcal{E}$ be a 2-cover of $\mathcal{E}$. Then, by section $3.4 \gamma$ induces an automorphism $\bar{\alpha}$ of $\mathcal{S}^{\prime}$. If the automorphism $\bar{\alpha}$ fixes the line $L$, then there exist exactly two automorphisms $\widetilde{\alpha}$ of $\mathcal{S}$ which extend the automorphism $\bar{\alpha}$ of $\mathcal{S}^{\prime}$.

Proof. As by Chapter 8 of [10] all the lines of $\mathcal{S}^{\prime}$ concurrent with $L$ are regular, it follows that all the lines of $\mathcal{S}^{\prime}$ are regular; see 1.3 .6 of [10]. Hence the generalized quadrangle $\mathcal{S}^{\prime}$ is isomorphic to the classical generalized quadrangle $Q(4, s)$; see 5.2 .1 of [10].

We will construct an automorphism $\widetilde{\alpha}$ of $\mathcal{S}$ such that $\bar{\alpha}$ is the restriction of $\widetilde{\alpha}$ to $\mathcal{S}^{\prime}$.

Choose a point $z$ of $\mathcal{A}$ and choose a line $N$ of $\mathcal{S}$ incident with $z$; the point of $\mathcal{S}^{\prime}$ incident with $N$ is denoted by $x^{*}$. Assume that $x^{*}$ is not on $L$. The point $z$ covers an ovoid $\mathcal{O}(z)$ of $\mathcal{S}^{\prime}$ and $\mathcal{O}(z)$ is subtended by the points $z^{\prime}, z^{\prime \prime}$ of $\mathcal{S}$. Let $z^{\widetilde{\alpha}}=z^{\prime}$. Then the line $N^{\widetilde{\alpha}}$ is the line $N^{\prime}=z^{\prime} x$ with $x=x^{* \widetilde{\alpha}}=x^{* \bar{\alpha}}$. For any line $T$ of $\mathcal{S}^{\prime}$, we define $T^{\widetilde{\alpha}}=T^{\bar{\alpha}}$.

Now let $y$ be a point of $z x^{*}$, with $x^{*} \neq y \neq z$. The point $y$ covers an ovoid $\mathcal{O}(y)$ of $\mathcal{S}^{\prime}$ and $\mathcal{O}(y)$ is subtended by a unique point $y^{\prime}$ of $N^{\prime}$; let $y^{\prime}=y^{\widetilde{\alpha}}$. Next, consider a point $v$ of $\mathcal{A}$, which is not collinear with $x^{*}$. Let $V$ be the line incident with $v$ and concurrent with $N$, let $w^{*}$ be the common point of $V$ and $\mathcal{S}^{\prime}$, and let $a$ be the common point of $N$ and $V$. The point $v$ covers an ovoid $\mathcal{O}(v)$ of $\mathcal{S}^{\prime}$ and $\mathcal{O}(v)$ is subtended by a point $v^{\prime}$ of the line $V^{\prime}=w a^{\widetilde{\alpha}}$ (note that this is indeed a line), with $w^{* \widetilde{\alpha}}=w$. Let $V^{\prime}=V^{\widetilde{\alpha}}$ and $v^{\prime}=v^{\widetilde{\alpha}}$. Then $\widetilde{\alpha}$ is defined for all points of $\mathcal{S}$ not collinear with $x^{*}$, for all points of the line $N$, for all points of $\mathcal{S}^{\prime}$, for the line $N$, for all lines concurrent with $N$ but not containing $x^{*}$, and for all lines of $\mathcal{S}^{\prime}$; for all these points and lines $\widetilde{\alpha}$ preserves incidence.

Each line $U$ concurrent with $L$, with $L$ consisting of translation points of $\mathcal{S}$, is regular. Let $U$ be a line of $\mathcal{S}$ concurrent with $L$, not concurrent with $N$, and not contained in $\mathcal{S}^{\prime}$. Let $u^{*}$ be the common point of $U$ and $L$. The line $U$ of $\mathcal{A}$ covers a rosette consisting of $s$ ovoids $\mathcal{O}\left(u_{1}\right), \mathcal{O}\left(u_{2}\right), \ldots, \mathcal{O}\left(u_{s}\right)$ of $\mathcal{S}^{\prime}$, with $u_{1}, u_{2}, \ldots, u_{s}$ the points of $\mathcal{A}$ incident with $U$. These ovoids have as common point $u=u^{* \widetilde{\alpha}}$, with $u$ on $L$. The ovoid $\mathcal{O}\left(u_{i}\right)$ is subtended by points $u_{i}^{1}, u_{i}^{2}$ of lines $U_{1}, U_{2}$ incident with $u$, with $i=1,2, \ldots, s$. The points $u_{i}$ which are not collinear with $x^{*}$ are mapped by $\widetilde{\alpha}$ onto points $u_{i}^{\widetilde{\alpha}}$, where $u_{i}^{\widetilde{\alpha}}$ is incident with a line $U_{1}, U_{2}$. Assume, by way of contradiction, that for at least one such $u_{k}$ we have that $u_{k}^{\widetilde{\alpha}}$ is incident with $U_{1}$, and that for at least one such $u_{l}$ we have that $u_{l}^{\widetilde{\alpha}}$ is incident with $U_{2}$. Let $\bar{U}_{k}$ be the line incident with $u_{k}$ and concurrent with $N$, and let $\bar{U}_{l}$ be the line incident with $u_{l}$ and concurrent with $N$. Let $\bar{u}_{k}$ be the common point of $\bar{U}_{k}$ and $\mathcal{S}^{\prime}$, and let $\bar{u}_{l}$ be the common point of $\bar{U}_{l}$ and $\mathcal{S}^{\prime}$. Consider also the elements $\bar{U}_{k}^{\widetilde{\alpha}}, \bar{U}_{l}^{\widetilde{\alpha}}, \bar{u}_{k}^{\widetilde{\alpha}}, \bar{u}_{l}^{\widetilde{\alpha}}$; the lines $\bar{U}_{k}^{\widetilde{\alpha}}, \bar{U}_{l}^{\widetilde{\alpha}}$ are concurrent with $N^{\widetilde{\alpha}}$.

First assume that $x^{*}$ and $u^{*}$ are collinear. By the regularity of $U$ the line $A$ incident with $\bar{u}_{l}$ and concurrent with $x^{*} u^{*}$, intersects the line $\bar{U}_{k}$. As $A$ is a line of $\mathcal{S}^{\prime}$, it contains the point $\bar{u}_{k}$. Hence $\bar{u}_{l} \sim \bar{u}_{k}$. It follows that $\bar{u}_{l}^{\widetilde{\alpha}} \sim \bar{u}_{k}^{\widetilde{\alpha}}$, and that $x u$ is concurrent with $\bar{u}_{l}^{\widetilde{\alpha}} \bar{u}_{k}^{\widetilde{\alpha}}$. The lines $x u, U_{1}, U_{2}$ are regular, hence the lines $x u, \bar{u}_{l}^{\widetilde{\alpha}} \bar{u}_{k}^{\widetilde{\alpha}}, N^{\widetilde{\alpha}}, \bar{U}_{k}^{\widetilde{\alpha}}, \bar{U}_{l}^{\widetilde{\alpha}}, u_{k}^{\widetilde{\alpha}} u=U_{1}, u_{l}^{\widetilde{\alpha}} u=U_{2}$ belong to a common grid. Consequently $u_{k}^{\widetilde{\alpha}} u=U_{1} \sim \bar{U}_{l}^{\widetilde{\alpha}}$ and $u_{l}^{\widetilde{\alpha}} u=U_{2} \sim \bar{U}_{k}^{\widetilde{\alpha}}$. So there arises a triangle with sides $U_{1}, U_{2}, \bar{U}_{l}^{\widetilde{\alpha}}$, a contradiction. It follows that we may assume that $x^{*} \nsim u^{*}$.

There exists a translation $\theta$ of $\mathcal{S}$ with translation point on $L$, which maps $u^{*}$ onto $u$, and which maps $u_{k}$ onto $u_{k}^{\widetilde{\alpha}}$. Then $\widetilde{\alpha}^{-1} \theta$ fixes the point $u$, the point $u_{k}^{\widetilde{\alpha}}$ and induces an isomorphism from $\mathcal{S}^{\prime}$ onto $\mathcal{S}^{\prime}{ }^{\theta}$. The point $u$ is a translation point of the generalized quadrangle $\mathcal{S}$. We may put $\mathcal{S}=\mathbf{T}(\mathcal{O})$, with $\mathcal{O}$ a generalized ovoid of $\mathbf{P G}(4 n-1, q), s=q^{n}$, and with $u$ the point $\infty$ of $\mathbf{T}(\mathcal{O})$. The subquadrangle $\mathcal{S}^{\prime}$ is a $\mathbf{T}\left(\mathcal{O}^{\prime}\right)$, with $\mathcal{O}^{\prime} \subset \mathcal{O}$ a generalized conic containing $L$ in $\mathbf{P G}(3 n-1, q)$ (see Chapter 7 of [15]); the subquadrangle $\mathcal{S}^{\prime}{ }^{\theta}$ is a $\mathbf{T}\left(\mathcal{O}^{\prime \prime}\right)=$ $T\left(\mathcal{O}^{\prime}\right)^{\theta}$, with $\mathcal{O}^{\prime \prime} \subset \mathcal{O}$ a generalized conic containing $L$. The lines $U_{1}=U^{\theta}, U_{2}$ are elements of $\mathcal{O} \backslash \mathcal{O}^{\prime}$. The line $N^{\theta}$ is an $n$-dimensional space containing an element $\widetilde{N}^{\theta} \in \mathcal{O} \backslash \mathcal{O}^{\prime}, \widetilde{N}^{\theta} \neq U_{1}, U_{2}$, the line $N^{\widetilde{\alpha}}$ is an $n$-dimensional space containing an element $\widetilde{N}^{\widetilde{\alpha}} \in \mathcal{O} \backslash \mathcal{O}^{\prime}, \widetilde{N}^{\widetilde{\alpha}} \neq U_{1}, U_{2}$. We have $N^{\theta} \cap \mathcal{S}^{\prime \theta}=\left\{x^{* \theta}\right\}$ and $N^{\widetilde{\alpha}} \cap \mathcal{S}^{\prime}=\{x\}$ (the point $x$ belongs to the space $\mathbf{P G}(3 n, q)$ containing $\mathcal{S}^{\prime}$, and the point $x^{* \theta}$ belongs to the space $\mathbf{P G}(3 n, q)^{\theta}$ containing $\mathcal{S}^{\prime \theta}$ ). The lines $\bar{U}_{k}^{\theta}, \bar{U}_{l}^{\theta}$ are $n$-spaces containing $U^{\theta}=U_{1} \in \mathcal{O}$, and they intersect $\mathcal{S}^{\prime \theta}$ in points $\bar{u}_{k}^{\theta}, \bar{u}_{l}^{\theta}$. If $\left\langle N^{\theta}, U^{\theta}\right\rangle \cap \mathbf{P G}(3 n-1, q)=\pi_{1}$, with $\pi_{1}(n-1)$-dimensional, then $\bar{u}_{k}^{\theta}, \bar{u}_{l}^{\theta}, x^{* \theta}$ are contained in an $n$-space $\widetilde{\pi}_{1}$ containing $\pi_{1}$ (as $\bar{U}_{k}^{\theta}$ and $\bar{U}_{l}^{\theta}$ intersect $N^{\theta}$ ). The lines $\bar{U}_{k}^{\widetilde{\alpha}}, \bar{U}_{l}^{\widetilde{\alpha}}$ are $n$-spaces containing $U_{1}, U_{2} \in \mathcal{O}$, and they intersect $\mathbf{P G}(3 n, q)$ in points $\bar{u}_{k}^{\widetilde{\alpha}}, \bar{u}_{l}^{\widetilde{\alpha}}$. If $\left\langle N^{\widetilde{\alpha}}, U_{1}\right\rangle \cap \mathbf{P G}(3 n-1, q)=\zeta_{1}$ and $\left\langle N^{\widetilde{\alpha}}, U_{2}\right\rangle \cap \mathbf{P G}(3 n-1, q)=\zeta_{2}$, then $\zeta_{1} \cap \zeta_{2}=\emptyset,\left\langle\zeta_{1}, \bar{u}_{k}^{\widetilde{\alpha}}\right\rangle=\widetilde{\zeta}_{1},\left\langle\zeta_{2}, \bar{u}_{l}^{\widetilde{\alpha}}\right\rangle=\widetilde{\zeta}_{2}$ and $\widetilde{\zeta}_{1} \cap \widetilde{\zeta}_{2}=\{x\}$ (as $\bar{U}_{k}^{\widetilde{\alpha}}$ and $\bar{U}_{l}^{\widetilde{\alpha}}$ intersect $N^{\widetilde{\alpha}}$ ). Considering all the points $u_{r}$ of $\mathcal{S} \backslash \mathcal{S}^{\prime}$ on $U$, which are not collinear with $x^{*}$, there arise in $\mathbf{T}(\mathcal{O}) s-1$ points $\bar{u}_{r}^{\theta}$ of $\widetilde{\pi}_{1} \backslash\left(\pi_{1} \cup\left\{x^{* \theta}\right\}\right)$. 
Then the isomorphism $\theta^{-1} \widetilde{\alpha}$ of $\mathcal{S}^{\prime}$ onto $\mathcal{S}^{\prime}{ }^{\theta}$ maps these points onto $s-1$ points $\bar{u}_{r}^{\widetilde{\alpha}}$ of $\left(\widetilde{\zeta}_{1} \cup \widetilde{\zeta}_{2}\right) \backslash\left(\zeta_{1} \cup \zeta_{2} \cup\{x\}\right)$; also, these points are not all in either $\widetilde{\zeta}_{1}$ or $\widetilde{\zeta}_{2}$. Notice also that $\left(x^{* \theta}\right)^{\theta^{-1} \widetilde{\alpha}}=x$. Clearly, this yields a contradiction. Hence the $s$ points of $U$ not collinear with $x^{*}$ are mapped by $\widetilde{\alpha}$ onto $s$ points of one line $U_{i}, i \in\{1,2\}$; this line is denoted by $U^{\widetilde{\alpha}}$. It follows that $\widetilde{\alpha}$ is defined for all lines $U$ concurrent with $L$.

Let $U$ be a line concurrent with $L$, but not concurrent with $N$ (possibly $U=L$ ). Consider the grid $\mathcal{G}$ containing $U$ and $N$, and let $V$ be a line of $\mathcal{G}$ not in $\mathcal{S}^{\prime}$ and not concurrent with $N$ or $U$. Let $x_{1}, x_{2}, \ldots, x_{s}$ be the points of $V$ not collinear with $x^{*}$, and let $X_{1}, X_{2}, \ldots, X_{s}$ be the lines of $\mathcal{G}$ concurrent with $N$, with $x_{i}$ incident with $X_{i}, i=$ $1,2, \ldots, s$. The lines $N^{\widetilde{\alpha}}, X_{1}^{\widetilde{\alpha}}, X_{2}^{\widetilde{\alpha}}, \ldots, X_{s}^{\widetilde{\alpha}}, U^{\widetilde{\alpha}}$ belong to a grid $\mathcal{G}^{\widetilde{\alpha}}$, and $x_{i}^{\widetilde{\alpha}}$ is incident with $X_{i}^{\widetilde{\alpha}}, i=1,2, \ldots, s$. The points $x_{1}^{\widetilde{\alpha}}, x_{2}^{\widetilde{\alpha}}, \ldots, x_{s}^{\widetilde{\alpha}}$ of the grid $\mathcal{G}^{\widetilde{\alpha}}$ belong to two concurrent lines $V_{1}, V_{2}$ (whose common point belongs to $\left.\mathcal{S}^{\prime}\right)$. It easily follows that $x_{1}^{\widetilde{\alpha}}, x_{2}^{\widetilde{\alpha}}, \ldots, x_{s}^{\widetilde{\alpha}}$ are incident with one of the lines $V_{1}, V_{2}$, say $V_{1}$. Let us put $V_{1}=V^{\widetilde{\alpha}}$.

Let $\mathcal{T}$ be the grid containing $N$ and $L$, and let $Y$ be a line containing a point $y$ of $\mathcal{T}$, with $x^{*}$ not incident with $Y$. We will show that the line $Y^{\widetilde{\alpha}}$ is uniquely defined. We may assume that $Y \nsim N$, that $Y \nsim L$, and that $Y \notin \mathcal{T}$ (putting $U=L$ in the previous paragraph, the images of all lines of $\mathcal{T}$ are well defined). Consider the grid $\mathcal{T}^{\prime}$ defined by $N$ and $Y$; notice that the pair $\{N, Y\}$ is regular as there is a (regular) line $U^{\prime}$ (containing $y$ ) intersecting $N, Y, L$. Let $u^{\prime}$ be the common point of $U^{\prime}$ and $L$. The grid $\mathcal{T}^{\prime}$ has a line $U^{\prime \prime}$ containing $u^{\prime}$, with $U^{\prime \prime} \nsim Y$. By the foregoing paragraph the line $Y^{\widetilde{\alpha}}$ is well defined.

Let $Y$ be a line not containing a point of $\mathcal{T}$ and not belonging to $\mathcal{S}^{\prime}$. Let $y$ be the common point of $Y$ and $\mathcal{S}^{\prime}$, let $Z$ be the line containing $y$ and concurrent with $L$, and let $Z \cap L=\{z\}$. Further, let $U$ be the line containing $z$ and concurrent with $N$. The grid defined by the regular line $U$ and the line $Y$ is denoted by $\mathcal{R}$. We determine the intersection of $\mathcal{R}$ and $\mathcal{S}^{\prime}$.

First assume that $x^{*} \chi z$. There are $s$ points $x_{1}, x_{2}, \ldots, x_{s}$ of $\mathcal{R}$ in $\mathcal{S}^{\prime}$, which are not incident with $Z$. The line $Z^{\prime}$ containing $x_{1}$ and concurrent with $Z$ belongs to $\mathcal{R}$ and $\mathcal{S}^{\prime}$, so contains the points $x_{1}, x_{2}, \ldots, x_{s}$. The line $Z^{\prime}$ does not contain $y$, as otherwise $\mathcal{R}$ contains at least three lines through $y$. The lines $Z, Z_{1}, Z_{2}, \ldots, Z_{s}$ of $\mathcal{R}$ concurrent with $U$ have well-defined images $Z^{\widetilde{\alpha}}, Z_{1}^{\widetilde{\alpha}}, Z_{2}^{\widetilde{\alpha}}, \ldots, Z_{s}^{\widetilde{\alpha}}$ as they have a point in common with $\mathcal{T}$. Also the regular line $U$ and the line $Z^{\prime}$, both belonging to $\mathcal{R}$, have well-defined images $U^{\widetilde{\alpha}}$ and $\left(Z^{\prime}\right)^{\widetilde{\alpha}}$. Clearly $U^{\widetilde{\alpha}}$ is also regular. Hence $Z^{\widetilde{\alpha}}, Z_{1}^{\widetilde{\alpha}}, Z_{2}^{\widetilde{\alpha}}, \ldots, Z_{s}^{\widetilde{\alpha}}, U^{\widetilde{\alpha}}$ and $\left(Z^{\prime}\right)^{\widetilde{\alpha}}$ belong to a grid $\mathcal{R}^{\widetilde{\alpha}}$. The images of the points of $Y$ are incident with lines $Y_{1}, Y_{2}$ and belong to $\mathcal{R}^{\widetilde{\alpha}}$; the common point of $Y_{1}, Y_{2}$ is the point $y^{\widetilde{\alpha}}$. It easily follows that these images are incident with just one of the lines $Y_{1}, Y_{2}$, say $Y_{1}$. So $Y^{\widetilde{\alpha}}=Y_{1}$ is well defined.

Next, assume that $x^{*} \sim z$. Then the intersection of $\mathcal{R}$ and $\mathcal{S}^{\prime}$ consists of the lines $U$ and $Z$. Let $W$ be a line of $\mathcal{S}^{\prime}$ through $y$, not containing $z$, and let $\mathcal{R}^{\prime}$ be a grid containing $W, Y$, and a point of $L$, but not containing $x^{*}$. Then all lines of $\mathcal{R}^{\prime}$ distinct from $Y$ have a well-defined image under $\widetilde{\alpha}$ and belong to a grid $\mathcal{R}^{\prime \widetilde{\alpha}}$. It easlly follows that $Y^{\widetilde{\alpha}}$ is well defined.

We have shown that $W^{\widetilde{\alpha}}$ is well defined for each line of $\mathcal{S}$ which does not contain $x^{*}$.

We have still to define $t^{\widetilde{\alpha}}$, with $t \sim x^{*}, t$ not incident with $N, t$ not in $\mathcal{S}^{\prime}$, and also $W^{\widetilde{\alpha}}$, with $x^{*}$ incident with $W$, $W \neq N, W$ not in $\mathcal{S}^{\prime}$. This is easy. Let $t$ be such a point. Let $E$ be a line containing $t$, with $E \neq t x^{*}$, and let $t^{\prime}$ be the point incident with $E^{\widetilde{\alpha}}$ and collinear with $x^{* \widetilde{\alpha}}=x$. Now let $E^{\prime}$ be a second line containing $t$, with $E^{\prime} \neq t x^{*}$, and let $t^{\prime \prime}$ be the point incident with $E^{\prime \widetilde{\alpha}}$ and collinear with $x$. Assume, by way of contradiction, that $t^{\prime} \neq t^{\prime \prime}$. If $E^{\widetilde{\alpha}}$ and $E^{\prime \widetilde{\alpha}}$ would be incident with a common point $\widehat{t}$, then, as $t^{\prime} \neq t^{\prime \prime}$, the point $\widehat{t}^{\widetilde{\alpha}^{-1}}$ would be incident with $E$ and $E^{\prime}$, with $\widetilde{t}^{\widetilde{\alpha}^{-1}} \neq t$, a contradiction. Let $D \sim E^{\widetilde{\alpha}}, D \sim E^{\prime \widetilde{\alpha}}, t^{\prime}$ not incident with $D$ and $t^{\prime \prime}$ not incident with $D$. Then $D^{\widetilde{\alpha}^{-1}} \sim E, D^{\widetilde{\alpha}^{-1}} \sim E^{\prime}, t$ not incident with $D^{\widetilde{\alpha}^{-1}}$. Hence there arises a triangle in $\mathcal{S}$, a contradiction. Consequently $t^{\prime}=t^{\prime \prime}$. Let us then put $t^{\prime}=t^{\widetilde{\alpha}}$.

Finally, let $x^{*}$ be incident with $W, W \neq N, W$ not in $\mathcal{S}^{\prime}$. Let $t$ be incident with $W, t \neq x^{*}$, and let $W^{\prime}=x t^{\widetilde{\alpha}}$. Let $r$ be incident with $W, r \neq x^{*}, r \neq t$, and let $W^{\prime \prime}=x r^{\widetilde{\alpha}}$. Assume, by way of contradiction, that $W^{\prime} \neq W^{\prime \prime}$ so that $r^{\widetilde{\alpha}} \not t^{\widetilde{\alpha}}$. Let $b \sim r^{\widetilde{\alpha}}, b \sim t^{\widetilde{\alpha}}, b \neq x$. Then $b^{\widetilde{\alpha}^{-1}} \sim r, b^{\widetilde{\alpha}^{-1}} \sim t$, with $b^{\widetilde{\alpha}^{-1}}$ not incident with $W$, clearly a contradiction. Let us then put $W^{\prime}=W^{\widetilde{\alpha}}$.

Now $\widetilde{\alpha}$ is defined for all points and lines of $\mathcal{S}$ and preserves incidence. Hence $\widetilde{\alpha}$ is an automorphism of $\mathcal{S}$ such that $\bar{\alpha}$ is the restriction of $\widetilde{\alpha}$ to $\mathcal{S}^{\prime}$.

If in the second paragraph of the proof the point $z^{\widetilde{\alpha}}$ is chosen to be $z^{\prime \prime}$, then the second such automorphism $\widetilde{\alpha}$ is obtained. 
Before proceeding, recall the next theorem.

Theorem 6.2 (K. Thas [19, 15]). Let $\mathcal{S}$ be a thick generalized quadrangle of order $\left(s, s^{2}\right)$ having a line $L$ of translation points and a subquadrangle $\mathcal{S}^{\prime}$ of order $s$ containing $L$, with the property that every subtended ovoid in $\mathcal{S}^{\prime}$ is doubly subtended. Then, for $s$ even $\mathcal{S}$ is classical and for $s$ odd $\mathcal{S}$ is a Kantor-Knuth generalized quadrangle.

Remark 6.3. [15] If $\mathcal{S}$ is a nonclassical Kantor-Knuth generalized quadrangle of order $\left(s, s^{2}\right)$, then any subquadrangle of order $s$ contains the unique line $L$ of translation points.

Theorem 6.4. Let $\mathcal{S}$ be a nonclassical Kantor-Knuth generalized quadrangle, let $L$ be the line of translation points, let $\mathcal{S}^{\prime}$ be a subquadrangle containing $L$ and assume that every subtended ovoid in $\mathcal{S}^{\prime}$ is doubly subtended. If $\gamma: \mathcal{A} \longmapsto \mathcal{E}$ is a cover of $\mathcal{E}$ with $\mathcal{A}=\mathcal{S} \backslash \mathcal{S}^{\prime}$, then the corresponding automorphism $\bar{\alpha}$ of $\mathcal{S}^{\prime}$ fixes the line L; also each automorphism of $\mathcal{E}$ fixes $L$.

Proof. The points of the geometry $\mathcal{E}$ are subtended ovoids of the subquadrangle $\mathcal{S}^{\prime} \cong Q(4, s)$. Each such ovoid is a translation ovoid relative to some point on $L$. It follows that the automorphism $\bar{\alpha}$ of $\mathcal{S}^{\prime}$ induced by $\gamma$ fixes the line $L$.

Theorem 6.5. Let $\mathcal{S}$ and $\mathcal{S}^{\prime}$ satisfy (i), (ii), (iii) as in Theorem 6.1] Then the cover $\pi: \mathcal{A} \longmapsto \mathcal{E}$ has the higher decomposition property.

Proof. If $\mathcal{S}$ is classical, then by [14] $\pi$ has the higher decomposition property. Hence suppose that $\mathcal{S}$ is not classical, so that by Theorem 6.2 $s$ is odd and $\mathcal{S}$ is a Kantor-Knuth generalized quadrangle. Let $\gamma: \mathcal{A} \longmapsto \mathcal{E}$ be any cover of $\mathcal{E}$. Then by Theorem 6.4 the corresponding automorphism $\bar{\alpha}$ of $\mathcal{S}^{\prime}$ fixes the line $L$ of translation points. By Theorem 6.1 the automorphism $\bar{\alpha}$ can be extended in two ways to an automorphism $\widetilde{\alpha}$ of $\mathcal{S}$ for which $\gamma=\pi \circ \widetilde{\alpha}$. Consequently $\pi$ has the higher decomposition property.

Corollary 6.6. Each automorphism $\alpha$ of the semi partial geometry $\mathcal{E}$ arising from $\pi$ is induced by exactly two automorphisms of the geometry $\mathcal{A}$.

Theorem 6.7. Let $\sigma \neq 1$ be the companion automorphism of the nonclassical Kantor-Knuth generalized qudrangle S. Let $\mathcal{S}^{\prime}$ be a subquadrangle of order $s$ with $\mathcal{S}^{\prime} \in \Omega_{1}$.

- If $\sigma^{2}=1$, then $\operatorname{Aut}(\mathcal{E})=\operatorname{Aut}\left(\mathcal{S}^{\prime}\right)_{L}$ and $|\operatorname{Aut}(\mathcal{A})|=2|\operatorname{Aut}(\mathcal{E})|$.

- If $\sigma^{2} \neq 1$, then $2|\operatorname{Aut}(\mathcal{E})|=\left|\operatorname{Aut}\left(\mathcal{S}^{\prime}\right)_{L}\right|$ and $|\operatorname{Aut}(\mathcal{A})|=2|\operatorname{Aut}(\mathcal{E})|$.

Proof. By [11] $|\operatorname{Aut}(\mathcal{S})|=(s+1)(s-1)^{2} s^{6} h \delta$, with $s=p^{h}$ and $p$ prime, $\delta=2$ for $\sigma^{2} \neq 1$ and $\delta=4$ for $\sigma^{2}=1$. The orbit $\Omega_{1}$ of subquadrangles $\mathcal{S}^{\prime}$ of order $s$ with 2-subtended ovoids has size $2 s^{2}$; see [14]. Hence $|\operatorname{Aut}(\mathcal{A})|=|\operatorname{Aut}(\mathcal{S})| / 2 s^{2}$. So $|\operatorname{Aut}(\mathcal{A})|=(s-1)^{2}(s+1) s^{4} h \delta / 2$. By Corollary 6.6 we have $2|\operatorname{Aut}(\mathcal{E})|=|\operatorname{Aut}(\mathcal{A})|$, so $|\operatorname{Aut}(\mathcal{E})|=(s-1)^{2}(s+1) s^{4} h \delta / 4$.

Also $\left|\operatorname{Aut}\left(\mathcal{S}^{\prime}\right)_{L}\right|=h s^{4}(s-1)\left(s^{2}-1\right)$; see $\S 4.6 .2$ of [22]. Consequently, for $\sigma^{2}=1$ we have $\operatorname{Aut}\left(\mathcal{S}^{\prime}\right)_{L}=\operatorname{Aut}(\mathcal{E})$, and for $\sigma^{2} \neq 1$ we have $2|\operatorname{Aut}(\mathcal{E})|=\left|\operatorname{Aut}\left(\mathcal{S}^{\prime}\right)_{L}\right|$.

Alternative proof of Theorem 6.1 and Corollary 6.6 in the case of $\sigma^{2}=1, \sigma \neq 1$.

Assume that $\sigma^{2}=1, \sigma \neq 1$. Then $\left|\operatorname{Aut}(\mathcal{S})_{\mathcal{S}^{\prime}}\right|=|\operatorname{Aut}(\mathcal{A})|=2\left|\operatorname{Aut}\left(\mathcal{S}^{\prime}\right)_{L}\right|$, the latter equality arising by merely comparing the explicit values of $|\operatorname{Aut}(\mathcal{A})|=\left|\operatorname{Aut}(\mathcal{S})_{\mathcal{S}^{\prime}}\right|$ (when $\sigma^{2}=1, \sigma \neq 1$ ) and $2\left|\operatorname{Aut}\left(\mathcal{S}^{\prime}\right)_{L}\right|$. As any subtended ovoid of $\mathcal{S}^{\prime}$ is doubly subtended we have that $|\operatorname{Aut}(\mathcal{A})| \leq 2|\operatorname{Aut}(\mathcal{E})|$ and, by Theorem 6.4 we have $\left|\operatorname{Aut}\left(\mathcal{S}^{\prime}\right)_{L}\right| \geq$ $|\operatorname{Aut}(\mathcal{E})|$, that is $\left.|\operatorname{Aut}(\mathcal{E})| \leq \mid \operatorname{Aut}\left(\mathcal{S}^{\prime}\right)_{L}\right)|=| \operatorname{Aut}(\mathcal{A})|/ 2 \leq| \operatorname{Aut}(\mathcal{E}) \mid$. Consequently $\operatorname{Aut}\left(\mathcal{S}^{\prime}\right)_{L}=\operatorname{Aut}(\mathcal{E})$ and $|\operatorname{Aut}(\mathcal{A})|=2|\operatorname{Aut}(\mathcal{E})|$. This proves Theorem 6.1 and Corollary 6.6 for $\sigma^{2}=1, \sigma \neq 1$.

\section{EMBEDDED KANTOR-KNUTH OVOIDS}

Let $\mathcal{Q}$ and $\mathcal{O}_{x}$ be as in section 4, Put $A:=\operatorname{Aut}(\mathcal{Q}) \cong \operatorname{Aut}(\mathcal{Q}(4, q))$. Then each element of $\mathcal{K}(\mathcal{Q}):=\mathcal{O}_{x}^{A}$ is, by definition, also called Kantor-Knuth ovoid. 


\subsection{The embedding category. Now consider an embedding}

$$
\gamma: \mathcal{Q}(4, q) \hookrightarrow \Gamma
$$

which we see as an isomorphism $\gamma: \mathcal{Q}(4, q) \mapsto \mathcal{Q}$, where $\mathcal{Q}$ is contained in $\Omega_{1} \cup \Omega_{2}$ - say $\mathcal{Q} \in \Omega_{1}$ w. 1 . 0 . g. Let $\mathcal{O}(\mathcal{Q})$ be the set of subtended Kantor-Knuth ovoids in $Q$ from points of $\Gamma \backslash \mathcal{Q}$ (and we use this notation throughout). We call $\gamma^{-1}(\mathcal{O}(2))$ the set of embedded ovoids of $Q(4, q)$ with respect to the embedding $\gamma$. Now we define a category $\operatorname{Emb}(Q(4, q), \Gamma)$ in which objects are diagrams of the above form

$$
\gamma: \mathcal{Q}(4, q) \rightarrow \mathcal{Q}
$$

and in which a morphism between objects $\gamma: \mathcal{Q}(4, q) \rightarrow \mathcal{Q}$ and $\gamma^{\prime}: \mathcal{Q}(4, q) \rightarrow \mathcal{Q}^{\prime}$ (where $\mathbb{Q}^{\prime} \in \Omega$ ) is a pair $(\alpha, \beta)$ of generalized quadrangle morphisms $\alpha: \mathcal{Q}(4, q) \rightarrow \mathcal{Q}(4, q)$ and $\beta: \mathcal{Q} \rightarrow Q^{\prime}$ such that the following diagram commutes:

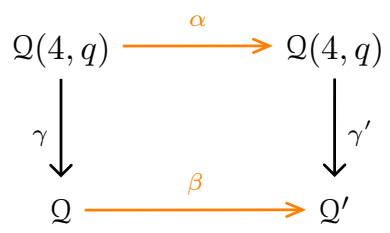

and such that $\beta(\mathcal{O}(Q)) \subseteq \mathcal{O}\left(Q^{\prime}\right)$. Without the latter property, the category would not detect the initial embeddings of the type given in (8): take for instance one flag $(u, U)$ in the first $2(4, q)$ on the top row, and define $\alpha$ by mapping all flags of $Q(4, q)$ on $(u, U)$. Define $\beta$ by mapping all flags of $Q$ to $\left(\gamma^{\prime}(u), \gamma^{\prime}(U)\right)$.

Since $\gamma$ and $\gamma^{\prime}$ are isomorphisms, the morphism $\beta$ also is an isomorphism if and only if $\alpha$ is.

The identity element of the object $\gamma: \mathcal{Q}(4, q) \rightarrow \mathcal{Q}$ is $\left(\operatorname{id}_{\mathcal{Q}(4, q)}, \operatorname{id}_{\mathcal{Q}}\right)$.

Later, we will show that we can relax the definition of morphism quite a bit, as follows: $(\alpha, \beta)$ is as above, but we ask that there is some $\mathcal{O} \in \mathcal{O}(Q)$ such that $\beta(\mathcal{O}) \in \mathcal{O}\left(Q^{\prime}\right)$ (instead of the entire action on $\mathcal{O}(Q)$ ), and that some special line for $\mathcal{O}$ is mapped to an appropriate special line for $\beta(\mathcal{O})$.

Note also that initially, $\alpha$ and $\beta$ not necessarily are isomorphisms. Still, we will show (in Corollary 7.4) that they have to be.

First we need some more theory.

A geometrical hyperplane in a thick GQ $\mathcal{S}$ of order $(s, t)$ is a subgeometry $\mathcal{S}^{\prime}$ with the property that each line $U$ of $\mathcal{S}$ either contains one point of $\mathcal{S}^{\prime}$, and then $U$ is not a line of $\mathcal{S}^{\prime}$, or all its points are points of $\mathcal{S}^{\prime}$, and then $U$ is a line of $\mathcal{S}^{\prime}$. It is easy to show that there only are three types of geometrical hyperplanes in this setting:

A $\mathcal{S}^{\prime}$ is an ovoid of $\mathcal{S}$ (and so $\mathcal{S}^{\prime}$ does not have lines);

B $\mathcal{S}^{\prime}$ is the point-line geometry of a set $x^{\perp}$, with $x$ a point of $\mathcal{S}$ (so the only lines of $\mathcal{S}^{\prime}$ are those lines of $\mathcal{S}$ incident with $x$ );

$\mathrm{C} \mathcal{S}^{\prime}$ is a subGQ of order $(s, t / s)$. This is the typical finite formulation of this case; in the general case, we want to say that $\mathcal{S}^{\prime}$ is a proper full subGQ which contains at least one point of every line in $\mathcal{S}$. Note that such a subGQ is maximal in the following sense: there is no proper subGQ $\widehat{\mathcal{S}}$ of $\mathcal{S}$ which properly contains $\mathcal{S}^{\prime}$. (Suppose by way of contradiction that such a $\widehat{\mathcal{S}}$ exists. Let $u$ be a point in $\widehat{\mathcal{S}} \backslash \mathcal{S}^{\prime}$, and let $U$ be any line of $\mathcal{S}$ incident with $u$. Then $U$ is incident with some point $v$ of $\mathcal{S}^{\prime}$. As $v$ is also a point of $\widehat{\mathcal{S}}$, it follows that $U$ is a line of $\widehat{\mathcal{S}}$. So $\widehat{\mathcal{S}}$ is both full and ideal, and hence coincides with $\mathcal{S}$ by [22, proposition 1.8.2].)

In the light of the previous discussion, we now show the next result (Theorem 7.3). First we observe an easy property.

Observation 7.1. If $\Gamma$ is a thick $G Q$ with an ovoid $\mathcal{O}$, and $\Gamma^{\prime}$ is a subGQ which contains $\mathcal{O}$, then $\Gamma=\Gamma^{\prime}$. 
Proof. Consider any line $U$ of $\Gamma^{\prime}$. Let $u \mathbf{I} U$ be arbitrary in $\Gamma$, but not contained in $\mathcal{O}$. Let $V \mathbf{I} u, V \neq U$. Then $V$ contains a point $\omega$ of $\mathcal{O}$. Since $V$ is the unique line in $\Gamma$ incident with $\omega$ and concurrent with $U$, it follows that $u \in \Gamma^{\prime}$, and so $\Gamma^{\prime}$ is full. Now let $v \in \Gamma^{\prime}$ and not contained in $\mathcal{O}$. Then any line on $v$ contains a point of $\mathcal{O}$, so such a line must be a line of $\Gamma^{\prime}$. It follows that besides full, $\Gamma^{\prime}$ is also ideal, so $\Gamma^{\prime}=\Gamma$ by [22, proposition 1.8.2].

We also recall an interesting result of Pasini [8], which generalizes an older result of Hughes [6] (which is the case of projective planes).

Theorem 7.2 (Hughes-Pasini [6, 8]). Let $\alpha$ be a morphism from a thick (possibly infinite) generalized $m$-gon $\mathcal{E}$ to a thick (possibly infinite) generalized $m$-gon $\mathcal{E}^{\prime}$, with $m \geq 3$. If $\alpha$ is surjective, then either $\alpha$ is an isomorphism, or each element in $\mathcal{E}^{\prime}$ has an infinite fiber in $\mathcal{E}$.

As we will see later on, the thickness condition is crucial (as the statement is not true without that assumption).

Theorem 7.3. Let $\Delta$ and $\Delta^{\prime}$ be finite thick GQs, and let $\phi: \Delta \mapsto \Delta^{\prime}$ be a GQ morphism which surjectively maps a geometrical hyperplane $\mathcal{G}$ of $\Delta$ to a geometrical hyperplane $\mathcal{G}^{\prime}$ of $\Delta^{\prime}$ :

$$
\phi(\mathcal{G})=\mathcal{G}^{\prime} .
$$

Then $\phi$ is surjective if $\mathcal{G}^{\prime}$ is not of type $B$, and also if $\mathcal{G}^{\prime}$ is not thin if it is of type $C$.

Proof. First note that $\phi(\Delta)$ is a (possibly degenerate) GQ: if $x^{\prime}$ is a point of $\phi(\Delta)$ not incident with the line $Y^{\prime}$ in $\phi(\Delta)$, and $x \in \phi^{-1}\left(x^{\prime}\right), Y \in \phi^{-1}\left(Y^{\prime}\right)$, then $x$ is not incident with $Y$, and so there is a unique line $Z$ such that $x \mathbf{I} Z \sim Y$. So there is a unique line $Z^{\prime}=\phi(Z)$ in $\phi(\Delta)$ such that $x^{\prime} \mathbf{I}^{\prime} Z^{\prime} \sim Y^{\prime}$.

CASE 1. Before starting the proof, note that the image of a partial ovoid through a GQ morphism not necessarily is a partial ovoid. Example: map all flags of a GQ $\mathcal{S}$, with partial ovoid $\mathcal{O}$, to a flag of type $(v, V)$, where $V$ is a fixed line, and such that the image of $\mathcal{O}$ is not a singleton.

We now prove the statement for ovoids $\mathcal{O}=\mathcal{G}^{\prime}$. In that case, $\mathcal{G}$ also is an ovoid. Observe that $\phi(\Delta)$ is a subGQ of $\Delta^{\prime}$ which contains $\mathcal{G}^{\prime}$; as $\mathcal{G}^{\prime}$ is an ovoid of the thick GQ $\Delta^{\prime}$, Observation 7.1 shows that $\phi(\Delta)=\Delta^{\prime}$, and so $\phi$ is surjective.

CASE 2. Now suppose $\mathcal{G}^{\prime}$ is of type C. Then $\mathcal{G}^{\prime}$ is thick by assumption. Obviously $\mathcal{G}$ is also of type C. Let $x$ be any point in $\Delta \backslash \mathcal{G}$. First suppose that $\phi(x) \in \mathcal{G}^{\prime}$ for each such point. Then $\phi$ induces an epimorphism $\widehat{\phi}$ from the thick GQ $\Delta$ onto the thick GQ $\mathcal{G}^{\prime}$. By Theorem $7.2, \widehat{\phi}$ is an isomorphism, so that $\Delta$ and $\mathcal{G}^{\prime}$ have the same parameters. As $\widehat{\phi}$ induces an isomorphism from $\mathcal{G}$ to $\mathcal{G}^{\prime}$, also $\mathcal{G}$ and $\mathcal{G}^{\prime}$ have the same parameters. Hence $\mathcal{G}=\Delta$, a contradiction.

Finally, if for some point $x \in \Gamma \backslash \mathcal{G}$, we have that $\phi(x) \notin \mathcal{G}^{\prime}$, we find that by maximality of $\mathcal{G}^{\prime}, \Delta^{\prime}$ and $\phi(\Delta)$ must coincide.

Note. Now we give a counter example to Theorem 7.3 for $\mathcal{G}^{\prime}$ a thin subGQ of the GQ $\Delta^{\prime}$ of order 3 . Consider $\mathbf{T}(\mathcal{O})=\Delta$ in $\mathbf{P G}(3,3)$ with $\mathcal{O}=\left\{a_{1}, a_{2}, a_{3}, a_{4}\right\}$ a conic in the plane $\delta$. Let $\zeta \neq \delta$ be a plane in PG $(3,3)$ containing $a_{1}$ and $a_{2}$. Further, let $t_{i}$ be the tangent line of $\mathcal{O}$ at $a_{i}$, with $i=1,2,3,4$. By definition, the (thin) GQ $\mathcal{G}=\mathcal{G}^{\prime}$ consists of the lines of $\Delta$ in $\zeta$, the points of $\Delta$ in $\zeta$, the point $(\infty)$ and the planes tangent to $\mathcal{O}$ which contain $t_{1}$ or $t_{2}$. Also, let $\Delta^{\prime}$ be any GQ of order 3 intersecting $\Delta$ in $\mathcal{G}=\mathcal{G}^{\prime}$, or let $\Delta^{\prime}=\Delta$. Now we construct the morphism $\phi: \Delta \mapsto \Delta^{\prime}$. Let $a_{1} a_{3} \cap a_{2} a_{4}=\{r\}$. Each element of $\mathcal{G}$ is mapped onto itself, so $\phi$ maps surjectively $\mathcal{G}$ onto $\mathcal{G}^{\prime}$. If $m$ is a point of $\mathbf{P G}(3,3)$ not in $\delta \cup \zeta$, then $\phi(m)=r m \cap \zeta ; \phi\left(a_{3}\right)=a_{1}$ and $\phi\left(a_{4}\right)=a_{2}$; if $R$ is a line of $\mathbf{P G}(3,3)$ containing $a_{i}, i \in\{3,4\}$, but not contained in $\delta$, then $\phi(R)=r R \cap \zeta$; finally, if $\eta \neq \delta$ is a plane containing $t_{i}, i \in\{3,4\}$ - say $i=3$, containing the point $\ell$ of $\mathbf{P G}(3,3) \backslash \delta$, then $\phi(\eta)$ is the plane $t_{1} \phi(\ell)$.

In a forthcoming paper, we hope to handle Theorem 7.3, CASE 2 without further finiteness and/or thickness restrictions. 
A groupoid is a category in which each morphism is an isomorphism. The core $\operatorname{cor}(\mathcal{C})$ of a category $\mathcal{C}$ is the maximal groupoid contained in the category as a subcategory.

Corollary 7.4. We have that $\operatorname{cor}(\operatorname{Emb}(2(4, q), \Gamma))=\operatorname{Emb}(2(4, q), \Gamma)$.

Proof. Consider a morphism $(\alpha, \beta)$. Since $\beta$ sends subtended ovoids of $Q$ to subtended ovoids of $Q^{\prime}$, we have by Theorem 7.3 that $\beta$ is surjective. Hence bijective, since we consider finite generalized quadrangles. So $\alpha$ is an isomorphism as well, and hence $\operatorname{Emb}(2(4, q), \Gamma)$ is a groupoid.

Two natural questions arise:

DT If $\mathcal{G}$ is a geometrical hyperplane of $\Delta$, and $\mathcal{G}^{\prime}$ is a geometrical hyperplane of $\Delta^{\prime}$, can $\phi(\mathcal{G})$ have a different type than $\mathcal{G}$ ?

$\mathrm{TB}$ What happens if $\mathcal{G}^{\prime}$ is of type $\mathrm{B}$ ?

It turns out that the answer to the second question is, that the statement of the previous theorem in case $\mathcal{G}^{\prime}$ is of type $B$, is not true, and that the answer of the first question is "yes."

We return to question TB. We keep using the notation of Theorem 7.3 and allow infinite generalized quadrangles $\Delta$ and $\Delta^{\prime}$, and let $\mathcal{G}^{\prime}$ be of type B. We also suppose that $\mathcal{G}$ is of type B. Suppose $\gamma$ is a surjective morphism from the geometry $x^{\perp}=\mathcal{G}$ to the geometry of $\underline{\gamma}(x)^{\perp}=\mathcal{G}^{\prime}$. Note that, if $\Delta$ has (possibly infinite) parameters $(u, v)$ and $\Delta^{\prime}$ parameters $\left(u^{\prime}, v^{\prime}\right)$, then as soon as $u \geq u^{\prime}$ and $v \geq v^{\prime}$, such $\underline{\gamma}$ are easy to construct. The condition is also necessary. Now let $w$ be any point of $\Delta$ not collinear with $x$; define $\gamma(w):=\underline{\gamma}(x)$. Let $W$ be any line incident with $w$, and let $\operatorname{proj}_{x}(W)=: W^{\prime}$; then define $\gamma(W)=\underline{\gamma}\left(W^{\prime}\right)$. For any point or line $X$ in $\mathcal{G}$, let $\gamma(X):=\underline{\gamma}(X)$. Then $\gamma$ is a well-defined morphism $\gamma: \Delta \mapsto \Delta^{\prime}$ which surjectively maps $\mathcal{G}$ to $\mathcal{G}^{\prime}$.

We also see that Theorem 7.2 is not true for thin quadrangles $\mathcal{E}^{\prime}$ (as $\mathcal{E}=\Delta$ can be taken finite).

Now we turn to question DT. Now let $\mathcal{G}$ be of type C, and of order $(u, v)$. Let $\mathcal{G}^{\prime}$ be of type B, and let the parameters of $\Delta^{\prime}$ be $\left(u^{\prime}, v^{\prime}\right)$. Suppose that $u \geq u^{\prime}, v \geq v^{\prime}$, and construct $\gamma: \mathcal{G} \mapsto \Delta^{\prime}$ as above, where $x$ is any point of $\mathcal{G}$. We will now extend $\gamma$ to a morphism $\bar{\gamma}: \Delta \mapsto \Delta^{\prime}$ with image $\mathcal{G}^{\prime}$, and thus answer our first question affirmatively.

First of all, $\bar{\gamma}$ coincides with $\gamma$ on $\mathcal{G}$. For $w$ a point in $\Delta$ not contained in $\mathcal{G}$, send $w$ to $\gamma(x)$. Now let $W$ be a line incident with $w$. If $W$ meets some line $W^{\prime}$ which is incident with $x$, then $\bar{\gamma}(W):=\gamma\left(W^{\prime}\right)$. If $W$ is not incident with such a line, choose $\bar{\gamma}(W)$ arbitrarily on $\gamma(x)$.

It is easy to see that $\bar{\gamma}$ is a morphism $\bar{\gamma}: \Delta \mapsto \Delta^{\prime}$ such that

$$
\bar{\gamma}(\Delta)=\bar{\gamma}(\mathcal{G})=\mathcal{G}^{\prime}
$$

7.2. The category $\widetilde{\operatorname{Emb}}(\mathcal{Q}(4, q), \Gamma)$. Note that a priori, section 7.1 is just one possible approach to the "embedding category": we could also have considered as objects the diagrams

$$
\mathcal{Q}(4, q) \stackrel{\gamma}{\longrightarrow} \mathcal{Q} \stackrel{\iota_{1}}{\longrightarrow} \Gamma
$$

with $\iota_{1}$ the canonical embedding of $\mathcal{Q}$ into $\Gamma$, where morphisms between objects $Q(4, q) \stackrel{\gamma}{\longrightarrow} \mathcal{Q} \stackrel{\iota_{1}}{\longrightarrow} \Gamma$ and $\mathcal{Q}(4, q) \stackrel{\gamma^{\prime}}{\longrightarrow} \mathcal{Q}^{\prime} \stackrel{\iota_{2}}{\longrightarrow} \Gamma$ would be 3-tuples $(\alpha, \beta, \delta)$ of generalized quadrangle morphisms $\alpha: \mathcal{Q}(4, q) \mapsto \mathcal{Q}(4, q)$, $\beta: \mathcal{Q} \mapsto \mathcal{Q}^{\prime}$ and $\delta: \Gamma \mapsto \Gamma$ such that the diagram 


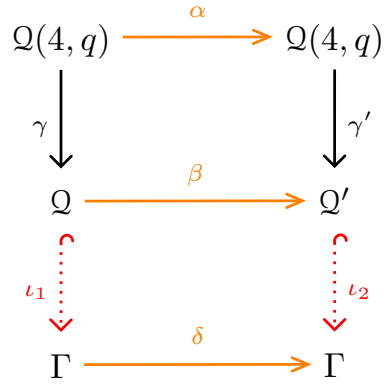

commutes. Note that $\alpha$ is an isomorphism if and only if $\beta$ is, and that if $\delta$ is an isomorphism (the case which is for us the most important one), it induces an isomorphism $\underline{\delta}: \iota_{1}(\mathcal{Q}) \mapsto \iota_{2}\left(\mathcal{Q}^{\prime}\right)$, so that $\beta$ and $\alpha$ also are isomorphisms. (If $\kappa=(\alpha, \beta, \delta)$ is an isomorphism, then there should be a $\kappa^{-1}$ such that $\kappa^{-1} \circ \kappa=\left(\mathrm{id}_{\mathcal{Q}(4, q)}, \mathrm{id}_{\mathcal{Q}}, \mathrm{id}_{\Gamma}\right)$. This can only happen if and only if $\alpha, \beta$ and $\delta$ are isomorphisms.)

The identity element of the object $Q(4, q) \stackrel{\gamma}{\longrightarrow} \mathcal{Q} \stackrel{\iota_{1}}{\longrightarrow} \Gamma$ is $\left(\operatorname{id}_{\mathcal{Q}(4, q)}, \operatorname{id}_{\mathcal{Q}}, \mathrm{id}_{\Gamma}\right)$.

We will call this second category $\widetilde{\operatorname{Emb}}(Q(4, q), \Gamma)$; later on, we will compare it with $\operatorname{Emb}(\mathcal{Q}(4, q), \Gamma)$.

As we will later see, a very important natural question arises: to understand the (iso)morphisms between objects in $\operatorname{Emb}(Q(4, q), \Gamma)$.

7.3. Special lines. Consider a $\mathcal{Q}(4, q)$-quadrangle $\mathcal{Q}$, and let $\mathcal{O}$ be a nonclassical Kantor-Knuth ovoid. Then $\mathcal{O}$ has a special point which is fixed by $\operatorname{Aut}(\mathcal{O})=\operatorname{Aut}(\mathcal{Q})_{\mathcal{O}}$, say $x$. The group $\operatorname{Aut}(\mathcal{O})$ acts transitively on the points of $\mathcal{O} \backslash\{x\}$. Suppose that

$$
\gamma_{i}: \mathcal{Q} \mapsto \mathcal{Q}_{i}^{\prime}
$$

is an isomorphism of generalized quadrangles, with $Q_{i}^{\prime}$ a $Q(4, q)$-quadrangle in a nonclassical Kantor-Knuth quadrangle $\Gamma$ in orbit $\Omega_{i}(i=1,2)$, such that $\gamma_{i}(\mathcal{O})$ is a subtended ovoid in $Q_{i}^{\prime}$. Let [ $\infty$ ] be the special line of $\Gamma$; then $[\infty] \mathbf{I} \gamma_{i}(x)$ and $[\infty]$ is a line of $Q_{i}^{\prime}$. Define $U_{i}:=\gamma_{i}^{-1}([\infty])$; it is a line incident with $x$. Then one can prove that the $\operatorname{Aut}(\mathcal{Q})_{\mathcal{O}}$-orbit which contains $U_{1}$ is different from the $\operatorname{Aut}(\mathcal{Q})_{\mathcal{O}}$-orbit which contains $U_{2}$; this will be done in the next section. It is important to note that as such we obtain two distinct orbits in 2 of lines incident with $x$, say $U_{1}(\mathcal{O}), U_{2}(\mathcal{O})$. Here, $U_{1}(\mathcal{O})$ denotes the lines coming from subGQs in the orbit $\Omega_{1}$, and $U_{2}(\mathcal{O})$ corresponds to subGQs in the orbit $\Omega_{2}$. We also use the notation $U_{1}\left(\mathcal{O}^{\prime}\right), U_{2}\left(\mathcal{O}^{\prime}\right)$, if $\mathcal{O}^{\prime}=\gamma_{i}(\mathcal{O})$. Remark that $U_{1}$ and $U_{2}$ depend on the embedding $\gamma_{i}$, and that $U_{i}(\mathcal{O})$ only depends on the choice of the orbit $\Omega_{i}$.

Now consider any isomorphism

$$
\beta: \mathcal{Q} \mapsto \mathcal{Q}^{\prime \prime}
$$

with $Q^{\prime \prime}$ a $Q(4, q)$-quadrangle in $\Gamma$, such that $\beta(\mathcal{O})$ is a subtended ovoid in $\mathbb{Q}^{\prime \prime}$. Then $\beta^{-1}([\infty]) \in U_{1}(\mathcal{O}) \cup U_{2}(\mathcal{O})$.

Remark 7.5. Later, in section 8 , we will see that $\left|U_{1}(\mathcal{O})\right| \equiv 0 \bmod 2$ if $\sigma^{2}=$ id.

7.4. Intrinsic way to recover the embedding. Given a fixed element of $\operatorname{Emb}(Q(4, q), \Gamma)$, there is an intrinsic way to recover the set $\mathcal{O}(Q)$, once we know at least one element of $\mathcal{O}(Q)$.

Put $C:=\operatorname{Aut}(\Gamma)_{\mathcal{Q}}$. Each line in $[\infty]^{\perp} \cap Q$ is an axis of symmetry (see section 4.1); denote the group generated by the symmetries defined by these lines by $L=L_{[\infty]}$. Note that $L \leq C$.

Now suppose $x \mathbf{I}[\infty]$ is an arbitrary point, and let $y, z$ be external points to $Q$ which are contained in $x^{\perp}$. If they are collinear, obviously there is an element of $L$ mapping $y$ to $z$. Suppose they are not collinear, so that $y x=: U$ and $z x=: V$ are different lines. Now consider a $\mathcal{Q}(4, q)$-subGQ $\widetilde{Q}$ containing $[\infty], U$ and $V$, such that $\mathcal{Q} \cap \widetilde{Q}$ is a grid $\mathcal{G}$ with parameters $(q, 1)$ (note that each of the $q-1 \mathcal{Q}(4, q)$-subGQs distinct from $Q$ containing $[\infty], U$ and $V$ meets $Q$ in such a grid (since they do not share all the lines on $x$ )). Let $M$ and $N$ be different lines in $\mathcal{G} \cap[\infty]^{\perp}$. Let $L(M, N)$ denote the subgroup of $L$ generated by the symmetries about $M$ and $N$; this group is 
isomorphic to $\mathrm{SL}_{2}(q)$ by [17, 18], stabilizes $\mathcal{Q}$ and $\widetilde{Q}$, and acts transitively on the points of $\mathcal{Q}$, respectively $\widetilde{Q}$, which are not contained in $\mathcal{G}$. In particular, there is an element in $L(M, N)$ which sends $y$ to $z$.

Since $L(M, N) \leq L$, and since $L$ acts transitively on the points of [ $\infty$ ], it now easily follows that $L$ acts transitively on the points of $\Gamma$ outside 2 . Whence it acts transitively on the subtended Kantor-Knuth ovoids in 2 . So if $\mathcal{O}_{x}$ is such an ovoid, we have that

$$
\mathcal{O}_{x}{ }^{C}=\mathcal{O}_{x}{ }^{L} .
$$

Remark that the result holds for any element $\mathcal{Q}$ in $\Omega_{1} \cup \Omega_{2}$.

By the preceding subsection, we have the following result.

Theorem 7.6 (Intrinsic Kantor-Knuth embedding). Consider an embedding

$$
\gamma: \mathcal{Q} \mapsto \mathcal{Q}^{\prime}
$$

where $\mathcal{Q} \cong \mathcal{Q}(4, q)$ and $\mathcal{Q}^{\prime}$ is a subGQ of a nonclassical Kantor-Knuth quadrangle $\Gamma$ of order $\left(q, q^{2}\right)$. Let $\mathcal{O}$ be any Kantor-Knuth ovoid in $\mathcal{Q}$ such that $\gamma(\mathcal{O})$ is a subtended ovoid in $\mathcal{Q}^{\prime}$. Let $[\infty]$ be the special line in $\Gamma$, and put $\gamma^{-1}([\infty])=U \in U_{1}(\mathcal{O}) \cup U_{2}(\mathcal{O})$. Then

$$
\gamma^{-1}\left(\mathcal{O}\left(Q^{\prime}\right)\right)=\mathcal{O}^{L_{U}}
$$

We still need to obtain the result mentioned at the end of the previous section, that is, that $U_{1}^{\operatorname{Aut}(\mathscr{Q})_{\mathcal{O}}} \neq U_{2}^{\operatorname{Aut}(\mathscr{Q})_{\mathcal{O}}}$. We use the notation of that section. By the results of the present section, it follows that $\left|\mathcal{O}\left(Q_{i}^{\prime}\right)\right|=\left|\mathcal{O}^{L_{U_{i}}}\right|$; but as $i$ differs, this quantity also changes by a factor 2 . It follows that there is no element in $\operatorname{Aut}(\mathcal{Q})_{\mathcal{O}}$ which maps $U_{1}$ to $U_{2}$.

7.5. Remark: configuration of special lines. We keep using the notation of the previous section.

Relative to one given embedding $\gamma$, one might wonder how the configuration of special lines corresponding to the embedded Kantor-Knuth ovoids looks like. The answer is very simple (and we take $U_{1}:=\gamma^{-1}([\infty])$ ).

Consider the set $\bigcup_{\mathcal{O} \in \gamma^{-1}\left(\mathcal{O}\left(\mathcal{Q}^{\prime}\right)\right)}\left(U_{1}(\mathcal{O}) \cup U_{2}(\mathcal{O})\right)$ with $\mathcal{Q}^{\prime} \in \Omega_{1}$; then by the fact that $L_{U_{1}}$ acts on $\gamma^{-1}\left(\mathcal{O}\left(\mathcal{Q}^{\prime}\right)\right)$, we have:

$$
\bigcup_{\mathcal{O} \in \gamma^{-1}\left(\mathcal{O}\left(Q^{\prime}\right)\right)}\left(U_{1}(\mathcal{O}) \cup U_{2}(\mathcal{O})\right)=U_{1}^{\perp}
$$

Also, $\bigcup_{\mathcal{O} \in \gamma^{-1}\left(\mathcal{O}\left(\mathcal{Q}^{\prime}\right)\right)} U_{2}(\mathcal{O})=U_{1}^{\perp} \backslash\left\{U_{1}\right\}$.

7.6. An equivalence relation on the Kantor-Knuth orbit. Let $\mathbb{F}_{q}$ be a finite field for $q$ an odd prime power, and let $\mathcal{Q}(4, q)$ be an orthogonal quadrangle in projective 4 -space. Let $\Omega(q, \sigma)$ be the complete Aut $(\mathcal{Q}(4, q))$-orbit of Kantor-Knuth ovoids of type $\sigma \in \operatorname{Aut}\left(\mathbb{F}_{q}\right)^{\times}$. Take any element $\mathcal{O} \in \Omega(q, \sigma)$, pick $U_{1} \in U_{1}(\mathcal{O}), U_{2} \in U_{2}(\mathcal{O})$, and define

$$
\left\{\begin{array}{l}
\mathcal{A}_{1}:=\left\{\left(\eta(\mathcal{O}), \eta\left(U_{1}\right)\right) \mid \eta \in \operatorname{Aut}(\mathcal{Q}(4, q))\right\} \\
\mathcal{A}_{2}:=\left\{\left(\eta(\mathcal{O}), \eta\left(U_{2}\right)\right) \mid \eta \in \operatorname{Aut}(\mathcal{Q}(4, q))\right\} .
\end{array}\right.
$$

By the above, $\mathcal{A}_{1}$ and $\mathcal{A}_{2}$ are different (so disjoint) subsets of $\Omega(q, \sigma) \times \mathcal{L}$, where $\mathcal{L}$ is the line set of $Q(4, q)$. Note that the natural projection

$$
\mathcal{A}_{1} \cup \mathcal{A}_{2} \mapsto \mathcal{L}
$$

is surjective.

Obviously, $\mathcal{A}_{1}$ corresponds to subGQs in $\Omega_{1}$ in the Kantor-Knuth GQ $\Gamma(q, \sigma)$, and $\mathcal{A}_{2}$ corresponds to subGQs in the other orbit.

Now define an equivalence relation $\sim_{\epsilon}$ on $\mathcal{A}_{i}, i=1,2$, as follows: $(\mathcal{O}, U) \sim_{\epsilon}\left(\mathcal{O}^{\prime}, U^{\prime}\right)$ if and only if $U=U^{\prime}$ and $\mathcal{O} \in \mathcal{O}^{\prime L_{U^{\prime}}}$. Note that this indeed defines an equivalence relation on both $\mathcal{A}_{1}$ and $\mathcal{A}_{2}$. 
Observation 7.7. Each equivalence class of $\sim_{\epsilon} \subset \mathcal{A}_{i}$ exactly singles out the subtended ovoids in an isomorphic image of $Q(4, q)$ in $\Gamma=\Gamma(q, \sigma)$, and $i$ determines in which orbit the isomorphic image of $Q(4, q)$ is situated.

7.7. Embedding theorem. As of now, we will denote objects of $\operatorname{Emb}(Q(4, q), \Gamma)$ sometimes as $[Q(4, q), \gamma, \mathcal{Q}]$.

Theorem 7.8 (Embedding theorem). Consider two objects $A:=[\mathcal{Q}(4, q), \gamma, \mathcal{Q}]$ and $B:=\left[\mathcal{Q}(4, q), \gamma^{\prime}, \mathcal{Q}^{\prime}\right]$ in $\operatorname{Emb}(Q(4, q), \Gamma)$. Then there exists an isomorphism $\kappa=(\alpha, \beta): A \mapsto B$ if and only if $Q$ and $Q^{\prime}$ belong to the same $\operatorname{Aut}(\Gamma)$-orbit. In particular, if $A=B, \operatorname{Aut}(A) \cong \operatorname{Aut}(\mathcal{Q})_{\mathcal{O}(\mathcal{Q})}$.

Proof. Let $\kappa=(\alpha, \beta): A \mapsto B$ be an isomorphism; then $\beta(\mathcal{O}(Q))=\mathcal{O}\left(Q^{\prime}\right)$, so that if $U$ is any special line for $\mathcal{O} \in \mathcal{O}(Q)$, then $|\mathcal{O}(Q)|=\left|\mathcal{O}^{L_{U}}\right|=\left|\mathcal{O}^{L_{\beta(U)}}\right|=\left|\mathcal{O}\left(Q^{\prime}\right)\right|$. This can only happen if $\mathcal{Q}$ and $Q^{\prime}$ are in the same $\Gamma$-orbit.

The other direction is obvious.

Now let $A=B$, and consider $\operatorname{Aut}(A)$. We are looking for pairs of automorphisms $(\alpha, \beta)$ such that the diagram below commutes:

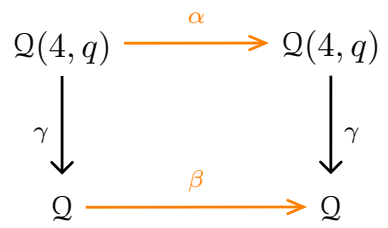

with $\beta(\mathcal{O}(2))=\mathcal{O}(Q)$.

Obviously, such pairs are completely and uniquely determined by the automorphisms $\beta$ of $Q$ which stabilize the set $\mathcal{O}(Q)$. This is what we had to prove.

7.8. Isomorphism classes of $\operatorname{Emb}(Q(4, q), \Gamma)$. Note that by Theorem $7.8, \operatorname{Emb}(Q(4, q), \Gamma)$ only has two isomorphism classes of objects. For essentially the same reason, the same is true for $\widehat{\operatorname{Emb}}(\mathcal{Q}(4, q), \Gamma)$.

7.9. The categories for different Kantor-Knuth quadrangles. Now let $\Gamma$ and $\Gamma^{\prime}$ be nonisomorphic KantorKnuth quadrangles of the same order $\left(q, q^{2}\right)$ - say with respective (different) associated $\mathbb{F}_{q}$-automorphisms $\sigma$ and $\sigma^{\prime}$. Question: $\operatorname{are} \operatorname{Emb}(Q(4, q), \Gamma)$ and $\operatorname{Emb}\left(Q(4, q), \Gamma^{\prime}\right)$ isomorphic?

Consider an object $A$ in $\operatorname{Emb}(Q(4, q), \Gamma)$; then by Theorem $7.8 \operatorname{hom}(A, A)=\operatorname{Aut}(A)$ can be identified with the automorphism group $\operatorname{Aut}(\mathcal{Q})_{\mathcal{O}(\mathcal{Q})}$ of $Q$ which fixes $\mathcal{O}(2)$ (this group automatically fixes $[\infty]$; for if some element

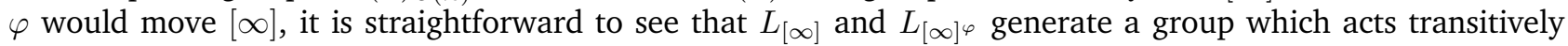
on the set of all lines, and this is sufficient information to force a contradiction: the set of special points of the ovoids in $\mathcal{O}(\mathcal{Q})$ coincides with the set of points incident with $[\infty]$, so $[\infty]$ must be fixed by $\left.\operatorname{Aut}(\mathcal{Q})_{\mathcal{O}(\mathcal{Q})}\right)$. As we have seen, this group acts transitively on the elements of $\mathcal{O}(\mathcal{Q})$. Note also that $\operatorname{hom}(A, A)$ is independent of the choice of $Q$ in $\Omega_{1}$ or $\Omega_{2}$. Since $\Gamma$ and $\Gamma^{\prime}$ are not isomorphic, $\sigma$ and $\sigma^{\prime}$ have a different order. Now if one of $\sigma$, $\sigma^{\prime}$ would be an involution, and the other is not, then $\operatorname{hom}(A, A)$ and $\operatorname{hom}(B, B)$ with $B$ in $\operatorname{Emb}\left(Q(4, q), \Gamma^{\prime}\right)$ are not isomorphic as abstract groups, since their sizes are different (as for instance $|\operatorname{hom}(A, A)|=|\mathcal{O}(\mathcal{Q})| \cdot|\operatorname{Aut}(\mathcal{O})|$, with $\mathcal{O} \in \mathcal{O}(Q)$ ). So the categories cannot be isomorphic. If we suppose that both $\sigma$ and $\sigma^{\prime}$ are involutions, then $\operatorname{hom}(A, A)$ and $\operatorname{hom}(B, B)$ do have the same size, so could be isomorphic in principle.

The following easy theorem reveals one crucial difference between the action of $\operatorname{Aut}(Q)_{\mathcal{O}(\mathcal{Q})}$ on $\mathcal{O}(\mathcal{Q})$ in case $\Gamma$ varies. First consider any nonclassical Kantor-Knuth ovoid $\mathcal{O}$ in $Q(4, q)$ with special automorphism $\sigma$, and let $u$ be its special point; furthermore, let $v \in \mathcal{O} \backslash\{u\}$. Then by definition, $H(u, v)$ is the subgroup of $\operatorname{Aut}(Q(4, q))_{\mathcal{O}}$ which fixes $u$ and $v$ linewise; we call this group kernel group or kernel of $\mathcal{O}$, and it is isomorphic to $\mathbb{F}_{\sigma}^{\times}$, where $\mathbb{F}_{\sigma}$ is the subfield of $\mathbb{F}_{q}$ fixed elementwise by $\sigma$ (see e. $g$. [1] for details). 
Theorem 7.9. Let $\Gamma$ be a nonclassical Kantor-Knuth $G Q$ of order $\left(q, q^{2}\right), Q \in \Omega_{1} \cup \Omega_{2}$ be any $Q(4, q)$-subGQ, and $\mathcal{O} \in \mathcal{O}(\mathcal{Q})$. Then $\operatorname{Aut}(\Gamma)_{\mathcal{Q}, \mathcal{O}(\mathcal{Q})}=\operatorname{Aut}(\Gamma)_{\mathcal{Q}}$ generates the kernel of $\mathcal{O}$. By "generates the kernel," we mean that the kernel group $H(u, v)$, with $u=[\infty] \cap \mathcal{O}$ and $v \in \mathcal{O} \backslash\{u\}$, of any ovoid $\mathcal{O} \in \mathcal{O}(\mathcal{Q})$ is contained in $\operatorname{Aut}(\Gamma)_{\mathcal{Q}, \mathcal{O}(\mathcal{Q})}$. (That is: $G(u, v) \cap \operatorname{Aut}(\Gamma)_{\mathcal{Q}, \mathcal{O}(\mathcal{Q})} \geq H(u, v)$, with $G(u, v)$ the kernel of the TGQ $\Gamma$.)

Proof. Let $\mathcal{O}$ be as in the statement, let $u=[\infty] \cap \mathcal{O}$ and let $v \in \mathcal{O} \backslash\{u\}$ be arbitrary. Consider the kernel $G(u, v)$ of the TGQ $\Gamma$ with respect to $u$ and $v$ (cf. [15, §3.4]). We have that $G(u, v)$ is isomorphic to $\mathbb{F}_{\sigma}^{\times}$(cf. [15, 4.7.3]). Then each element $\nu \in G(u, v)$ fixes each line of $Q$ incident with $u$ or $v$, so applying [18, Lemma 4.2.5] on $Q \cap Q^{\nu}$, we conclude that $Q$ is fixed by $\nu$. So $G(u, v)$ faithfully induces an automorphism group of $Q$ (faithfully because a nontrivial automorphism of $\Gamma$ that fixes $Q$ elementwise, cannot fix lines outside $Q$ ). Now let $w$ be any point in $\{u, v\}^{\perp} \backslash \mathcal{Q}$; then $G(u, v)$ fixes $\mathcal{O}_{w}=w^{\perp} \cap Q$. Suppose w. 1. o. g. that $\mathcal{O}=\mathcal{O}_{x}$, with $x$ a point of $\Gamma \backslash \mathcal{Q}$ subtending $\mathcal{O}_{x}$. Then $x \in\{u, v\}^{\perp}$, so if we put $w=x$, we deduce that $G(u, v)$ fixes $\mathcal{O}_{x}$. Whence $G(u, v)$ induces the entire kernel group $H(u, v) \cong G(u, v)$ of $\mathcal{O}_{x}$. The statement of the theorem follows.

Corollary 7.10. We have that $\operatorname{Aut}(\mathcal{Q})_{\mathcal{O}(\mathcal{Q})}$ generates the kernel of any such $\mathcal{O}$.

Proof. Immediate as $\operatorname{Aut}(\Gamma)_{\mathcal{Q}, \mathcal{O}(\mathcal{Q})} / N \leq \operatorname{Aut}(\mathcal{Q})_{\mathcal{O}(\mathcal{Q})}$, with $N$ the kernel of the action $\operatorname{Aut}(\Gamma)_{\mathcal{Q}, \mathcal{O}(\mathcal{Q})}$ on $\mathcal{Q}$.

Even if $\operatorname{hom}(A, A)$ and $\operatorname{hom}(B, B)$ would be isomorphic, the corresponding actions are not, as the following theorem shows.

Theorem 7.11. Let $\Gamma$ and $\Gamma^{\prime}$ be nonisomorphic Kantor-Knuth GQs of order $\left(q, q^{2}\right)$, and let $Q \leq \Gamma$ and $Q^{\prime} \leq \Gamma^{\prime}$ be $\mathrm{Q}(4, q)$-subGQs. Then the actions $\operatorname{Aut}(\mathrm{Q})_{\mathcal{O}(\mathcal{Q})} \curvearrowright \mathcal{O}(\mathcal{Q})$ and $\operatorname{Aut}(\mathcal{Q})_{\mathcal{O}\left(\mathcal{Q}^{\prime}\right)} \curvearrowright \mathcal{O}\left(\mathrm{Q}^{\prime}\right)$ are not equivalent.

Proof. Since $\Gamma$ and $\Gamma^{\prime}$ are defined by different automorphisms of Aut $\left(\mathbb{F}_{q}\right)$, the corresponding kernels are different subfields of $\mathbb{F}_{q}$. So if $\mathcal{O} \in \mathcal{O}(Q)$ and $\mathcal{O}^{\prime} \in \mathcal{O}\left(Q^{\prime}\right)$, they define different isomorphism classes of kernel groups. This implies the theorem.

7.10. A weird observation. Let $U$ be any line of $\mathcal{S}=Q(4, q)$, and define $L=L_{U}$ as the group generated by all symmetries with axis contained in $U^{\perp}$. Let $H(L)$ be the kernel group, with respect to the points $u$ and $v$, with $u \mathbf{I} U$ and $v \not u$, generated by $L$ in the sense of the previous sections: it is $G(u, v) \cap L$ with $G(u, v)$ the kernel of the TGQ $\mathcal{S}$ with translation point $(\infty)=u$ (and with respect to $v$ ).

We will prove the following result without any calculation, only using embedding theory.

Theorem 7.12. If $q$ is odd, $H(L)$ is a subgroup of $\mathbb{F}_{p}^{\times}$, where $q$ is a power of the prime $p$.

Proof. Suppose $q$ is odd (and fixed), and consider any embedding $\gamma: \mathcal{Q}(4, q) \mapsto \mathcal{Q}$, with $\mathcal{Q}$ a subquadrangle of a nonclassical Kantor-Knuth GQ $\Gamma$, and $\gamma(U)$ the line [ $\infty]$ of $\Gamma$. Since $L_{U}=\gamma^{-1}\left(L_{[\infty]}\right)$, and as $L_{U}$ and $H\left(L_{U}\right)$ are independent of $\Gamma$, we can consider all such embeddings. For a fixed $\gamma$, we have seen that the kernel group generated by $L_{[\infty]}$ is contained in the kernel of the Kantor-Knuth TGQ (once we have chosen a suitable translation point).

It easily follows that $H\left(L_{U}\right)$ is contained in the intersection of the kernel groups obtained by letting $\gamma$ (and so also $\Gamma$, and the automorphism $\sigma \in \operatorname{Aut}\left(\mathbb{F}_{q}\right)$ ) vary. So $H\left(L_{U}\right)$ must be a subgroup of $\mathbb{F}_{p}^{\times}=\bigcap_{\sigma \in \operatorname{Aut}\left(\mathbb{F}_{q}\right)} \mathbb{F}_{\sigma}^{\times}$.

\section{THE AUTOMORPHISM GROUP VIA THE GOOD ORBIT?}

Let $\mathcal{Q} \cong \mathcal{Q}(4, q)$ be any subGQ of order $(q, q)$ in a given GQ $\Gamma$ isomorphic to $\mathcal{Q}(5, q)$; then $\mathcal{E}$, the geometry of subtended ovoids and rosettes of ovoids, is 2-covered by $\mathcal{A}=\Gamma \backslash \mathcal{Q}$. Now it is a simple fact that

$$
\operatorname{Aut}(\Gamma)_{\mathcal{Q}} / N \cong \operatorname{Aut}(\mathcal{E}) \text {, }
$$

with $N$ the kernel of the action of $\operatorname{Aut}(\Gamma)_{\mathcal{Q}}$ on $\mathcal{Q}$, and that if $x$ is a point in $\Gamma \backslash \mathcal{Q}$, then $\operatorname{Aut}(\mathcal{Q})_{\mathcal{O}_{x}}$ is induced by $\operatorname{Aut}(\Gamma)_{Q, x}$. 
So we ask ourselves whether the same is true if we replace $\Gamma$ by a nonclassical Kantor-Knuth quadrangle, and $Q$ taken in the appropriate subGQ-orbit. Since the size of the subGQ-orbit $\Omega_{1}$ is rather small compared to the size of $\Omega_{2}$, and since each subGQ $Q \in \Omega_{1}$ is 2-covered by $\Gamma \backslash \mathcal{Q}$, it is a natural question to ask how big a part of the full automorphism group (in $Q$ ) of an element $\mathcal{O} \in \mathcal{O}(\mathcal{Q})$ is recovered by its automorphism group induced by $\operatorname{Aut}(\Gamma)_{\mathcal{Q}}$. Since the special line $[\infty]$ is fixed by $\operatorname{Aut}(\Gamma)$, we will be at least a factor 2 off as it is not fixed by $\operatorname{Aut}(\mathcal{Q})_{\mathcal{O}}$. Still, as we will see below in a simple calculation, the automorphism group induced by $\operatorname{Aut}(\Gamma)_{\mathcal{Q}}$ will be very large in comparison to $\operatorname{Aut}(\mathcal{O})$ : indeed, it will turn out to have half the size of $\operatorname{Aut}(\mathcal{Q})_{\mathcal{O}}$.

Keep using the notation as in the previous section. Since $\left|\Omega_{1}\right|=2 q^{2}$, it is easy to observe that the size of $\operatorname{Aut}(\Gamma)_{\mathcal{O}_{x}}=\left(\operatorname{Aut}(\Gamma)_{\mathcal{Q}}\right)_{\mathcal{O}_{x}}$ is

$$
\frac{(q+1)(q-1)^{2} q^{6} \delta h}{2 q^{2} \cdot\left(q^{2}(q+1)(q-1) / 2\right)}=(q-1) q^{2} \delta h .
$$

In $Q$, this group induces a group of size $(q-1) q^{2} \frac{\delta}{2} h$.

By a calculation in [11], the size of the complete automorphism group of $\mathcal{O}_{x}$ in $\operatorname{Aut}(Q)$ is $(q-1) q^{2} \cdot \delta h$. It is known that $\operatorname{Aut}\left(\mathcal{O}_{x}\right):=\operatorname{Aut}(\mathcal{Q})_{\mathcal{O}_{x}}$ fixes some point $u \in \mathcal{O}_{x}$ (recall section [5); let $U$ be any line incident with $u$, and put $\ell:=\left|U^{\operatorname{Aut}(\mathcal{Q})_{\mathcal{O}_{x}}}\right|$.

As $\operatorname{Aut}(\mathcal{Q})_{U}$ is isomorphic to $\mathbf{P}_{\mathbf{C}}(q)$ in its action on $U$, we know that $\left|\operatorname{Aut}(\mathcal{Q})_{U} / M\right|=(q+1) q(q-1) h$, with $M$ the kernel of the action of $\operatorname{Aut}(\mathcal{Q})_{U}$ on $U$ (the latter seen as a point set). So

$$
\left|\operatorname{Aut}\left(\mathcal{O}_{x}\right)\right| /\left(\left|U^{\operatorname{Aut}\left(\mathcal{O}_{x}\right)}\right| \cdot\left|M \cap \operatorname{Aut}\left(\mathcal{O}_{x}\right)\right|\right) \text { divides } q(q-1) h .
$$

Here,

$$
\frac{\left|\operatorname{Aut}\left(\mathcal{O}_{x}\right)\right|}{\left|U^{\operatorname{Aut}\left(\mathcal{O}_{x}\right)}\right|}=\left|\operatorname{Aut}\left(\mathcal{O}_{x}\right)_{U}\right|,
$$

and we have to mod out the trivial action of $M \cap \operatorname{Aut}\left(\mathcal{O}_{x}\right)$. Note that $\operatorname{Aut}\left(\mathcal{O}_{x}\right)_{U}$ is contained in $\operatorname{Aut}(\mathcal{Q})_{U, v}$, with $v=\mathcal{O} \cap U$.

We will use the following fact below:

Fact: let $Y$ be a full grid in a $2(4, q)$ with $q$ odd. Then the elementwise stabilizer of $Y$ in $\operatorname{Aut}(Q(4, q))$ has size 2 .

Consider a Sylow 2-subgroup $S_{2}$ of $M \cap \operatorname{Aut}\left(\mathcal{O}_{x}\right)$. Then $S_{2}$ fixes $U$ pointwise. Let $v \mathbf{I} U$ be any point incident with $U$; then by the fact that $q$ is odd, $S_{2}$ has to fix at least one other line $V$ incident with $v$ besides $U$. Each such line meets $\mathcal{O}_{x}$ in a point, which is necessarily fixed by $S_{2}$. As no two points of $\mathcal{O}_{x}$ are collinear, it easily follows that the fixed elements structure of the group $S_{2}$ is a full grid of order $(q, 1)$ if $S_{2} \neq\{\mathrm{id}\}$.

By the aforementioned fact, It follows that $\delta / 2$ divides $\ell=\left|U^{\operatorname{Aut}(\mathcal{Q})_{\mathcal{O}_{x}}}\right|$.

If $q$ is a square, and $\sigma^{2}=$ id $\neq \sigma$, then $\delta=4$, so each such orbit has size $\ell$ divisible by 2 . In case $U=[\infty], \ell=2$ is also the maximal size of such an orbit, since we know that the full automorphism group of $\mathcal{O}_{x}$ inside $Q$ has size $(q-1) q^{2} \delta h$, and since the action of $\operatorname{Aut}(Q)_{\mathcal{O}_{x}}$ on $\mathcal{O}_{x}$ is faithful. If $\sigma^{2} \neq$ id, then $\delta=2$, so the divisibility condition becomes vacuous. In any case, when $U=[\infty]$ we have that $\ell \leq 2$, since $\left(\operatorname{Aut}(\Gamma)_{\mathcal{Q}}\right)_{\mathcal{O}_{x}}$ induces an automorphism group of $\mathcal{O}_{x}$ inside $\mathcal{Q}$ which fixes $[\infty]$.

Comparing the different sizes. As we have seen, for $\mathcal{O}_{x}$ as above, we obtain that its stabilizer inside $(\operatorname{Aut}(\Gamma))_{Q}$ has size $(q-1) q^{2} \frac{\delta}{2} h$, just a factor 2 shy of the size of its full automorphism group. 


\section{REFERENCES}

[1] I. Bloemen, J. A. Thas and H. Van Maldeghem. Translation ovoids of generalized quadrangles and hexagons, Geom. Dedicata 72 (1998), 19-62.

[2] M. R. Brown. Semipartial geometries and generalized quadrangles of order $\left(r, r^{2}\right)$, in: Finite geometry and combinatorics (Deinze, 1997), Bull. Belg. Math. Soc. Simon Stevin 5 (1998), 187-205.

[3] I. Cardinali and N. S. N. Sastry. Elliptic ovoids and their rosettes in a classical generalized quadrangle of even order, Proc. Indian Acad. Sci. Math. Sci. 126 (2016), 591-612.

[4] J. De Kaey and H. Van Maldeghem. A characterization of the split Cayley generalized hexagon $H(q)$ using one subhexagon of order $(1, q)$, Discrete Math. 294 (2005), 109-118.

[5] J. W. P. Hirschfeld and J. A. Thas. General Galois Geometries, Springer Monographs in Mathematics.,Springer, London, 2016.

[6] D. R. Hughes. On homomorphisms of projective planes, 1960 Proc. Sympos. Appl. Math. Vol. 10, pp. 45?52, American Mathematical Society, Providence, R.I.

[7] B. Krim. Generalized Polygons with Doubly Transitive Ovoids, Ph. D. Thesis, Universität Stuttgart, 2013.

[8] A. Pasini. The nonexistence of proper epimorphisms of finite thick generalized polygons, Geom. Dedicata 15 (1984), $389-397$.

[9] S. E. Payne. Collineations of the generalized quadrangles associated with q-clans, Combinatorics '90 (Gaeta, 1990), pp. 449-461, Ann. Discrete Math. 52, North-Holland, Amsterdam, 1992.

[10] S. E. Payne and J. A. Thas. Finite Generalized Quadrangles. Second edition, EMS Series of Lectures in Mathematics, European Mathematical Society (EMS), Zürich, 2009.

[11] T. Penttila and B. Williams. Ovoids of parabolic spaces, Geom. Dedicata 82 (2000), 1-19.

[12] J. A. Thas. 3-regularity in generalized quadrangles: a survey, recent results and the solution of a longstanding conjecture, Combinatorics '98 (Mondello), Rend. Circ. Mat. Palermo (2) Suppl. No. 53 (1998), 199-218.

[13] J. A. Thas and S. E. Payne. Spreads and ovoids in finite generalized quadrangles, Geom. Dedicata 52 (1994), $227-253$.

[14] J. A. Thas and K. Thas. Covers of generalized quadrangles, Glasg. Math. J. 60 (2018), 585-601.

[15] J. A. Thas, K. Thas and H. Van Maldeghem. Translation Generalized Quadrangles, Series in Pure Mathematics 26, World Scientific Publishing Co. Pte. Ltd., Hackensack, NJ, 2006.

[16] K. Thas. Translation generalized quadrangles for which the translation dual arises from a flock, Glasg. Math. J. 45 (2003), $457-474$.

[17] K. Thas. The classification of generalized quadrangles with two translation points, Beiträge Algebra Geom. 43 (2002), 365-398.

[18] K. Thas. Symmetry in Finite Generalized Quadrangles, Frontiers in Mathematics, Birkhäuser Verlag, Basel, 2004.

[19] K. Thas. A stabilizer lemma for translation generalized quadrangles, European J. Combin. 28 (2007), 1-16.

[20] K. Thas. A Course on Elation Quadrangles, EMS Series of Lectures in Mathematics, European Mathematical Society (EMS), Zürich, 2012.

[21] K. Thas and H. Van Maldeghem.Geometric characterizations of finite Chevalley groups of type $B_{2}$, Trans. Amer. Math. Soc. 360 (2008), 2327-2357.

[22] H. Van Maldeghem. Generalized Polygons, Monographs in Mathematics 93, Birkhäuser Verlag, Basel, 1998.

Ghent University, Department of Mathematics, KrijgslaAn 281, S22 and S25, B-9000 Ghent, Belgium

E-mail address: thas.joseph@gmail.com; koen.thas@gmail.com 